\title{
Torsion D-Branes in
}

\section{Nongeometrical Phases}

\author{
Ilka Brunner $^{1}$ \\ Department of Physics and Astronomy \\ Rutgers University \\ Piscataway, NJ 08855 USA \\ ibrunner@physics.rutgers.edu \\ Jacques Distler ${ }^{2}$ \\ Theory Group, Physics Department \\ University of Texas at Austin \\ Austin, TX 78712 USA \\ distler@golem.ph.utexas.edu
}

\begin{abstract}
In a geometrical background, D-brane charge is classified by topological K-theory. The corresponding classification of D-brane charge in an arbitrary, nongeometrical, compactification is still a mystery. We study D-branes on non-simply-connected Calabi-Yau 3-folds, with particular interest in the D-branes whose charges are torsion elements of the K-theory. We argue that we can follow the $\mathrm{D}$-brane charge through the nongeometrical regions of the Kähler moduli space and, as evidence, explicitly construct torsion D-branes at the Gepner point in some examples. In one of our examples, the Gepner theory is a nonabelian orbifold of a tensor product of minimal models, and this somewhat exotic situation seems to be essential to the physics.
\end{abstract}

e-print archive: http://arXiv.org/hep-th/0102018

${ }^{1}$ Work supported in part DOE Grant DE-FG02-96ER40959.

${ }^{2}$ Work supported in part by NSF Grant PHY0071512 and the Robert A. Welch Foundation. 


\section{Introduction}

The D-branes that were analyzed thus far are mainly BPS branes preserving a fraction of the space-time supersymmetry. Starting with the work of Sen, it has been realized that there are also stable but non-BPS D-branes. The existence of (some of) these states has then been interpreted by Witten: Dbrane charges are classified by K-theory $[1,2]$ rather than cohomology. In this context, Sen's non-BPS states are stable because they carry a conserved discrete charge - a torsion element of the $\mathrm{K}$-theory ${ }^{1}$. There are two possible approaches to understand the physics of these stable non-BPS states: First, one can use a microscopic formulation, where D-branes are described as boundary conditions in a conformal field theory. Alternatively, one can use the language of $\mathrm{K}$-theory and vector bundles. Both of these approaches have been used to study toroidal orbifolds in the literature. In this paper, we study non-BPS D-branes in a geometrically more interesting background - Calabi-Yau compactifications whose K-theory has a torsion part. We are particularly interested in the dependence of the physics of torsion D-branes on the moduli of the closed string background.

In the large-radius limit, where geometrical reasoning applies, we know that D-brane charge is classified by topological K-theory. These geometrical concepts are no longer available in the stringy regime. Here, boundary conformal field theory provides a powerful tool for the investigation of stringy D-brane physics. While these methods are in principle applicable at generic points in moduli space, they are most useful at rational or "Gepner" points, where the theory is exactly solvable.

Still, while a detailed description of the D-branes may not be possible at a generic point in the moduli space, one might hope to achieve a cruder goal, namely the classification of the allowed D-brane charges. That is, we would like to define a "quantum K-theory" (the phrase appeared in [5], in analogy with quantum cohomology) which would classify the allowed Dbrane charges everywhere in the moduli space, and which would reduce to topological K-theory in the geometrical limit. And, wherever possible, we would like to make contact between it and the results of boundary conformal field theory.

Turning on a (flat) B-field leads rather naturally to a twisted version of the differential K-theory of $[6,7]$ (see also $[8,9]$ for earlier work). But to define our quantum K-theory, we would also need to understand how it

\footnotetext{
${ }^{1}$ Not all stable, non-BPS D-branes are explained this way. For instance, among the Dbranes of $[3,4]$ are ones which are stable in some region of the moduli space, for energetic reason, not because they carry a conserved charge.
} 
deforms when one turns on a finite value for the Kähler modulus.

While we won't be able to provide a definition of quantum K-theory, we will, at least, be able to describe how the quantum K-groups vary as we vary the moduli. More precisely, we will be able to determine the automorphism of the quantum $\mathrm{K}$-theory that results from traversing an incontractible cycle in the moduli space.

For the free part of the K-theory, these automorphisms are calculable using Mirror Symmetry. The comparison of BPS D-brane charges at different points in moduli spaces was studied in $[10,11,12,13,14,15,16,17,18,19]$. Our challenge is to extend these results to include the torsion in the K-theory. In the simplest class of examples (a more precise criterion will be discussed in §2.4), the automorphisms all act trivially on the torsion subgroup. So the torsion subgroup of the quantum K-theory is, in some sense, "constant" and independent of the moduli.

We won't address the following much harder question. Given a (torsion) class in the quantum K-theory at a point in the moduli space: is there a stable D-brane in the conformal field theory at that point representing the given quantum K-theory class? The analogous question for BPS branes is hard enough $[20,21,22]$.

We will see that, in some examples, it is possible to construct a stable brane in terms of boundary conformal field theory at the Gepner point, corresponding to a torsion class in the K-theory. This provides some evidence that there is, at least, a path between large-radius and the Gepner point along which the torsion branes are stable. It is not so clear that the torsion branes are stable near the conifold point (see §7.2).

We will present two examples: Both of them are quotients of the quintic by a freely-acting discrete group. The first is a quotient by a freely-acting $\mathbb{Z}_{5}$ scaling symmetry. The Kähler moduli space has three distinguished points: a "large-radius" point, a Gepner point, and a (mirror of a) conifold point. We construct torsion branes at large-radius and as bound states of BPS branes at the Gepner point. We find explicitly that the monodromies about these two points act trivially on the torsion subgroup of the quantum K-theory. Since there are only three boundary points of the Kähler moduli space, the monodromy about the conifold point must also act trivially on the torsion subgroup.

In the second example, we mod out by an additional orbifold action, which is a cyclic permutation of the five coordinates. This example was investigated by Aspinwall and Morrison [23]. They found that the tree-level 
topological string amplitudes (the chiral ring) have a $\mathbb{Z}_{5}$ symmetry, relating different points in the moduli space, which is not a symmetry of the full theory. In particular, the 1-loop topological string amplitudes distinguish these points. We find that the D-brane spectrum is also not invariant under this would-be $\mathbb{Z}_{5}$ symmetry. Rather, the CFTs at points in the moduli space related by this $\mathbb{Z}_{5}$ differ by turning on (topologically trivial) discrete torsion. This, of course, alters the spectrum of D-branes. We determine the monodromies (up to a certain ambiguity, involving the torsion) in the Kähler moduli space and, in particular, we determine which $\mathrm{D}$-branes become massless at each of the five points corresponding to (mirrors of a) "conifold"-type singularity.

Section $\S 2$ is devoted to laying out our guiding assumptions in determining the monodromies in the quantum K-theory as one moves about in the moduli space; subsections $\S 2.1, \S 2.2$ and $\S 2.5$ contain essential mathematical background to our computations. Sections $\S 3, \S 4$ review the essential conformal field theoretic background. In $\S 5$, we construct the BPS boundary states on the Gepner orbifolds we are interested in and in $\S 6$, we superpose them to form the desired torsion branes. $\S 7$ and $\S 8$ are devoted to our examples, and a detailed comparison of the conformal field theoretic and geometrical results.

\section{K-theory and D-branes}

We begin with a short review of some relevant facts about K-theory. Much of this material will be familiar to many readers; we include it here for completeness.

\section{$2.1 \quad$ D-brane charge}

In this paper we will be considering Type II string theory on spacetimes of the form $\mathbb{R}^{4} \times X$, where $X$ is a compact Calabi-Yau manifold. We will be interested in wrapped D-branes which correspond to particles in the noncompact $\mathbb{R}^{4}$. As has become familiar, $\mathrm{D}$-brane charge takes values in $\mathrm{K}$-theory, but precisely which $\mathrm{K}$-theory is relevant here? For D-branes which correspond to particles in $\mathbb{R}^{4}$, this is $K_{\widehat{c p t}}^{\bullet}\left(\mathbb{R}^{4} \times X\right)$, where by $K_{\widehat{c p t}}$, we mean compactly-supported in space, but not in time. Since the time-direction is contractible, this is

$$
K_{c p t}^{\bullet}\left(\mathbb{R}^{4} \times X\right)=K_{c p t}^{\bullet}\left(\mathbb{R}^{3} \times X\right)
$$


We can identify $K_{c p t}^{\bullet}(M)=K^{\bullet}(M, \partial M)$, the relative K-theory of $M$ with respect to its boundary. In our case, $\partial\left(\mathbb{R}^{3} \times X\right)=S^{2} \times X$. The relative $\mathrm{K}$-theory fits into a 6 -term exact sequence

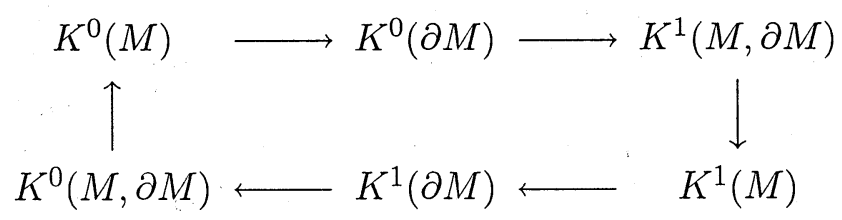

To evaluate the various terms in this sequence, we use the fact that if $K^{\bullet}(X)$ or $K^{\bullet}(Y)$ is freely-generated, there is a Künneth formula for $K^{\bullet}(X \times Y)$,

$$
\begin{aligned}
& K^{0}(X \times Y)=K^{0}(X) \otimes K^{0}(Y) \oplus K^{1}(X) \otimes K^{1}(Y) \\
& K^{1}(X \times Y)=K^{0}(X) \otimes K^{1}(Y) \oplus K^{1}(X) \otimes K^{0}(Y)
\end{aligned}
$$

In our case, $K^{0}\left(\mathbb{R}^{3}\right)=\mathbb{Z}, K^{0}\left(S^{2}\right)=\mathbb{Z}+\mathbb{Z}$ and $K^{1}\left(\mathbb{R}^{3}\right)=K^{1}\left(S^{2}\right)=0$. Plugging this information into (2.1), we have

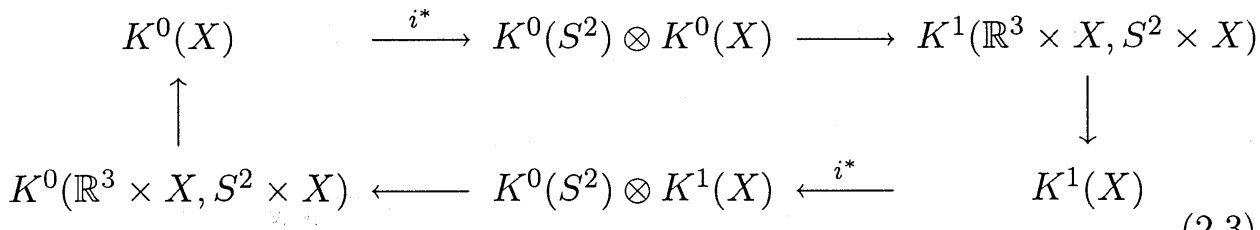

The maps $i^{*}$ are injective; they involve taking a class on $\mathbb{R}^{3}$ and restricting it to the 2-sphere at infinity. So $K^{1}\left(\mathbb{R}^{3} \times X, S^{2} \times X\right)=K^{0}\left(S^{2}\right) \otimes$ $K^{0}(X) / i^{*}\left(K^{0}(X)\right)=K^{0}(X)$ and similarly for $K^{0}$. So, for Type IIB, our $\mathrm{D}$-brane charge takes values in

$$
K_{c p t}^{0}\left(\mathbb{R}^{4} \times X\right) \simeq K^{1}(X)
$$

and for Type IIA, it takes values in

$$
K_{\widehat{c p t}}^{1}\left(\mathbb{R}^{4} \times X\right) \simeq K^{0}(X)
$$

This shift in degree should be familiar from ordinary E\&M. There, the charge density, $j \in \mathrm{H}_{\widehat{c p t}}^{3}\left(M_{3} \times \mathbb{R}\right)=\mathrm{H}_{c p t}^{3}\left(M_{3}\right)$ and by a similar exact sequence for relative cohomology, we find $\mathrm{H}_{c p t}^{3}\left(M_{3}\right) \simeq \mathrm{H}^{2}\left(\partial M_{3}\right) / i^{*}\left(\mathrm{H}^{2}\left(M_{3}\right)\right)$. This isomorphism is known as Gauss's Law; we compute the charge by integrating a 2-form, $* F$, over the boundary of a region of space. So we should regard $(2.4),(2.5)$ as the K-theoretic analogues of Gauss's Law. 


\subsection{The push-forward}

We will be particularly interested in the K-theory classes corresponding to Dbranes wrapped around submanifolds of $X$. Indeed, these will form a basis for the K-theory. The basic construction we will need is the K-theoretic push-forward. Given a submanifold $i: S \hookrightarrow X$, and a K-theory class $v$ on $S$, we can use the Thom homomorphism in K-theory to obtain a K-theory class, $i_{!} v$, on $X$. This class is characterized by the Atiyah-Hirzebruch Theorem.

Let $f: Y \rightarrow X$ be a continuous map between smooth, compact, connected manifolds. If $\operatorname{dim}(Y)-\operatorname{dim}(X)=0 \bmod 2$, then for each $a \in K^{0}(Y)$, there is a class $f_{!} a \in K^{0}(X)$ such that

$$
\operatorname{ch}\left(f_{!} a\right) \hat{A}(X)=f_{*}\left(\operatorname{ch}(a) e^{\frac{1}{2} d} \hat{A}(Y)\right)
$$

where $d \in \mathrm{H}^{2}(Y)$ is a class whose mod-2 reduction is $w_{2}(Y)-f^{*} w_{2}(X)$ and $f_{*}$ is the push-forward in cohomology. The definition of the push-forward, $f_{!}$, depends on a choice ${ }^{2}$ of the class $d$. If $Y, X$ are almost complex manifolds, there is a canonical choice for $d: \operatorname{set}^{3} d=c_{1}(Y)-f^{*} c_{1}(X)$ and (2.6) simplifies to

$$
\operatorname{ch}\left(f_{!} a\right) \operatorname{Td}(X)=f_{*}(\operatorname{ch}(a) \operatorname{Td}(Y))
$$

If $\operatorname{dim}(Y)-\operatorname{dim}(X)=1 \bmod 2$, then $f_{!} a \in K^{1}(X)$. Viewing $K^{1}(X)$ as a subgroup of $\tilde{K}^{0}\left(X \times S^{1}\right)$, we have the same formula (2.6), with $X$ replaced by $X \times S^{1}$ (so the difference in dimensions is again even).

In the presence of torsion, (2.7) (and similar formulæ that we use elsewhere) may not completely characterize the push-forward. In the dimension we are working, we can multiply by 6 to clear denominators. If (as will be true in our examples) there are no elements which are 2-torsion or 3-torsion, this operation has no kernel. The resulting equality would, in general, hold as an equality of integral Chern classes only modulo torsion. But, in our application, $Y$ is a complex submanifold of a Calabi-Yau 3-fold, $X$. The push-forward in K-theory is supported in a tubular neighbourhood of $Y$ For complex codimension 2 or 3 , the compactly-supported K-theory of the tubular neighbourhood (which is isomorphic to the K-theory of $Y$ ) is torsion-free, so the equality actually holds over the integers. In complex codimension 1 ( $Y$ a divisor on $X$ ), we can use the simpler result that $i_{!} \mathcal{O}_{Y}=L-\mathcal{O}$, where $L$ is the line bundle whose divisor is $Y$.

\footnotetext{
${ }^{2}$ There is an obstruction to defining the push-forward if no such class, $d$, exists. The implications of this for D-branes was explored in [24].

${ }^{3}$ This choice of $d$ implements the twisting of [25].
} 


\subsection{Monodromies}

We are, of course, not just interested in compactification on a particular Calabi-Yau manifold, $X$, but on a family of such manifolds, parametrized by some moduli space, $\mathcal{M}$. Over each point in $\mathcal{M}$, the allowed D-brane charges form a discrete abelian group, $\Gamma$. Locally, there is not much structure; since the charges are discrete, not much can happen to them. But if we circle a singular locus (the singular loci are complex-codimension one in $\mathcal{M}$ ), the group $\Gamma$ will come back to itself only up to an automorphism. So we have a $\Gamma$-bundle over the moduli space, and this bundle is characterized by a set of automorphisms $\in \operatorname{Aut}(\Gamma)$, one for each of the for each of the homotopicallynontrivial paths in $\mathcal{M}$.

If we mod out by the torsion in the $\mathrm{K}$-theory, the quotient $K(X) / K(X)_{\text {tor }}$ is a lattice and we have more structure. If we map $K(X) \rightarrow K(X, \mathbb{C})$, then the latter forms a complex (in fact, holomorphic) vector bundle over $\mathcal{M}$, with a flat connection (whose flat sections may be taken to be $K(X) / K(X)_{\text {tor }}$ ). The automorphisms of the lattice are just given by the holonomies of this connection and can be written as monodromy matrices with respect to a basis of flat sections. These are essential ingredients in defining the Special Geometry of $\mathcal{M}$.

On the level of $K(X) / K(X)_{t o r}$, the monodromies may be determined, rather explicitly, using Mirror Symmetry. Our task, however, will be to extend them to act on all of $K(X)$, including the torsion. For this purpose, it will prove more insightful to write topological (K-theoretic) formulæ. For this, we follow $[26,27]$.

We will mostly study the monodromies in the Kähler moduli space, and at that, we will mostly specialize to the case of one-dimensional Kähler moduli spaces. The monodromy around the large-radius limit is given shifting the $B$-field by the generator, $\xi$, of $\mathrm{H}^{2}(X)$. Since the B-field enters into the Chern character by

$$
C h(\mathcal{F})=\operatorname{Tr}\left(e^{F+B}\right)
$$

we see that shifting $B \rightarrow B+\xi$ corresponds to tensoring with a line bundle, $L$ such that $c_{1}(L)=\xi$.

Another singular locus of the moduli space is the (mirror of the) conifold, at which the volume of $X$ shrinks to zero size (while the volumes of 2-cycles and 4-cycles stay finite) [28]. In this case, the monodromy is [26, 27]

$$
v \mapsto v-k \operatorname{Ind}\left(\bar{\partial}_{v}\right) \mathcal{O}
$$

Here $v$ is an element of $K^{0}(X)$, and $\mathcal{O}$ is the trivial line bundle. In the case 
of hypersurfaces in toric varieties (as considered by [27]), the coefficient $k$ is 1. For the orbifolds we will consider later, we will find $k>1$.

\subsection{Pairings}

If all we had was the structure of an abelian discrete group (modulo torsion, a lattice), then the automorphisms could be rather more general. However, there are bilinear pairings on the K-theory, and we must demand that the automorphisms be symmetries of these pairings.

First, there is the usual intersection pairing,

$$
(., .): K^{0}(X) \times K^{0}(X) \rightarrow \mathbb{Z}
$$

This is a skew-symmetric bilinear pairing given by

$$
(v, w)=\operatorname{Ind}\left(\bar{\partial}_{v \otimes \bar{w}}\right)=\int_{X} \operatorname{ch}(v \otimes \bar{w}) T d(X)
$$

Normally, this is written in terms of the Dirac Index, rather than the Dolbeault Index. They agree on a Calabi-Yau manifold. It is skew-symmetric because $\operatorname{Ind}\left(\bar{\partial}_{\bar{v}}\right)=-\operatorname{Ind}\left(\bar{\partial}_{v}\right)$. It clearly annihilates $K_{\text {tor }}^{0}(X)$ and it is nondegenerate on $K^{0}(X) / K_{\text {tor }}^{0}(X)$.

There is a similar pairing on $K^{1}(X)$, given by the natural skew-symmetric map

$$
K^{1}(X) \times K^{1}(X) \rightarrow K^{0}(X)
$$

(where we have used Bott periodicity), followed by taking the index. Again, this is nondegenerate on $K^{1}(X) / K_{\text {tor }}^{1}(X)$.

These intersection pairings annihilate the torsion in K-theory. To capture information about the torsion, we can study the torsion-pairing [29, 30, 31]. This is a little more subtle to define, and we will review its definition and the relation to conformal field theory elsewhere [32]. For present purposes, it suffices that it is a nondegenerate pairing

$$
\langle., .\rangle ; K^{0}(X)_{t o r} \times K^{1}(X)_{t o r} \rightarrow \mathbb{R} / \mathbb{Z}
$$

In our examples (this is not the general situation!), $K^{1}(X)_{\text {tor }}$ is generated by D1-branes wrapped around torsion 1-cycles on $X^{4}$, and $K^{0}(X)_{t o r}$ is generated by elements of the form $L-\mathcal{O}$, for $L$ a flat line bundle on $X$. In this case,

\footnotetext{
${ }^{4}$ In general, these generate only a subgroup of $K^{1}(X)_{t o r}$, and similarly for $K^{0}(X)_{t o r}$.
} 
the torsion pairing is just the holonomy of the flat line bundle (corresponding to an element of $K^{0}(X)_{\text {tor }}$ ) around the torsion 1-cycle (corresponding to an element of $\left.K^{1}(X)_{t o r}\right)$.

Now, it is a fundamental feature of special geometry that the monodromies on the Kähler moduli space leave $K^{1}(X) / K^{1}(X)_{t o r}$ invariant, and similarly, the monodromies on the complex-structure moduli space leave $K^{0}(X) / K^{0}(X)_{t o r}$ invariant. So, if we ignore the torsion, we need only check the invariance of one or the other of the intersection pairings.

With the torsion, things are more subtle. If a monodromy on the Kähler moduli space acts as a nontrivial automorphism on $K^{0}(X)_{t o r}$, then, for the torsion pairing to be invariant, it must also act nontrivially on $K^{1}(X)_{\text {tor }}$ while leaving $K^{1}(X) / K^{1}(X)_{\text {tor }}$ invariant.

In our examples, we will, in fact, find that all of $K^{1}(X)$, including the torsion, is actually invariant under the monodromies of the Kähler moduli space, or equivalently that $K^{0}(X)_{\text {tor }}$ is invariant. At least for the example of $\S 7$, that will be justified a posteriori by our construction of the torsion Dbranes at the Gepner point. For the example of $\S 8$, we were unable to prove that the action on the torsion subgroup was trivial, but we were unable to find a nontrivial action consistent with all of our other requirements.

As we said, the monodromies must leave the intersection pairing invariant. That is,

$$
(M v, M w)=(v, w)
$$

This is obviously true for the large radius monodromy, since

$$
(v \otimes L, w \otimes L)=(v, w)
$$

for any line bundle $L$.

We also need to consider the effect on the torsion pairing. In the examples we consider, the ring structure of the K-theory will be such that, if $\alpha \in$ $K^{0}(X)_{\text {tor }}$, then $\alpha \otimes L=\alpha$ for any line bundle $L$, so that the monodromy acts trivially on $K^{0}(X)_{t o r}$. If we take the monodromy to act trivially on $K^{1}(X)$, we then have that both torsion subgroups are fixed by the monodromy, and hence (trivially) so is the torsion pairing. More interesting is a case in which $\alpha \otimes H=\alpha^{\prime} \neq \alpha$. In that case, where the monodromy acts as a nontrivial automorphism of $K^{0}(X)_{t o r}$. In order for the torsion pairing to be invariant, the monodromy would also have to act nontrivially on $K^{1}(X)_{t o r}$ (while still acting trivially on $\left.K^{1}(X) / K^{1}(X)_{t o r}\right)$.

Similarly the monodromy about the conifold (2.9) leaves the intersection 
pairing invariant. One can see this by writing the monodromy as

$$
v \mapsto v-k(v, \mathcal{O}) \mathcal{O}
$$

and using the skew-symmetry of the pairing.

This monodromy has a very simple interpretation. Let's say that at some singularity, a brane (a charged particle in the 4D effective theory) corresponding to K-theory class $w$ becomes massless. Circling the singularity shifts the $\theta$-angle - of the $U(1)$ for which this particle is electrically-charged - by $2 \pi$. By the Witten effect, the charge of a particle in K-theory class $v$ gets shifted by ${ }^{5}$

$$
v \mapsto v-(v, w) w
$$

when you shift $\theta \rightarrow \theta+2 \pi$. If $k$ such particles become massless, then the shift is (2.15). More generally, if several, mutually-local, particles become massless, the shift is

$$
v \mapsto v-\sum_{i}\left(v, w_{i}\right) w_{i}
$$

where mutual locality means $\left(w_{i}, w_{j}\right)=0$. As we mentioned in $\S 2.3$, at the conifold, it is the D6-brane, corresponding to the K-theory class $\mathcal{O}$ which becomes massless.

\subsection{The Atiyah-Hirzebruch and Cartan-Leray spectral se- quences}

In order to carry through our computations, we will need to be able to compute the cohomology of a Calabi-Yau manifold $X=Y / G$, given the knowledge of the cohomology of the covering space $Y$. And, given the cohomology of $X$, we will need to be able to compute its K-theory.

Two spectral sequences come to our aid here. The Cartan-Leray spectral sequence allows one to compute the homology of $X$. The Atiyah-Hirzebruch spectral sequence allows one to compute its K-theory. Both of these are spectral sequences of a double-complex [33].

For a cohomology spectral sequence (like AHSS), the $r^{\text {th }}$ approximation to the cohomology we are after is a bigraded complex, $E_{r}^{p, q}$, together with a

\footnotetext{
${ }^{5}$ Strictly speaking, this argument involving charges only fixes the action on $K^{0}(X) / K^{0}(X)_{t o r}$. However, the monodromy must also commute with the action of the quantum symmetry, to be discussed in $\S 2.6$. This, together with the action on the quotient, will frequently fix the actionon all of $K^{0}(X)$. Since this monodromy acts trivially on $K^{0}(X)_{t o r}$, the torsion pairing is preserved if it also acts trivially on $K^{1}(X)$.
} 
differential

$$
d_{r}: E_{r}^{p, q} \rightarrow E_{r}^{p+r, q-r+1}
$$

which increases the total degree, $n=p+q$, by one. The $(r+1)^{s t}$ approximation, $E_{r+1}$ is the cohomology of $d_{r}$ on $E_{r}$. Raoul Bott has likened spectral sequences to perturbation theory, each successive approximation getting closer and closer to the desired answer. In most applications, the $E_{r}^{p, q}$ vanish outside of some finite range in $p$ (or outside of some finite range in q). Consequently, unlike perturbation theory, the spectral sequence is guaranteed to converge after a finite number of steps to something we can call $E_{\infty}^{p, q}$. What the spectral sequence converges to, however, is not quite the cohomology we are interested in. Rather, it converges to the associated graded, $\operatorname{Gr}\left(\mathrm{H}^{\bullet}\right)$. That is, the cohomology group $\mathrm{H}^{n}$ has a filtration (sequence of subgroups, $F_{i}^{(n)}$ )

$$
\mathrm{H}^{n}=F_{0}^{(n)} \supset F_{1}^{(n)} \supset F_{2}^{(n)} \supset F_{3}^{(n)} \supset \ldots
$$

such that $F_{p}^{(n)} / F_{p+1}^{(n)}=E_{\infty}^{p, n-p}$. So, in general, even after we have computed $E_{\infty}$, we still have an extension problem to solve, in order to recover the cohomology groups $\mathrm{H}^{n}$ themselves.

A homology spectral sequence (like CLSS) is very similar. The $r^{\text {th }}$ approximation is a bigraded complex, $E_{p, q}^{r}$, but this time the differential lowers the total degree by one

$$
d_{r}: E_{p, q}^{r} \rightarrow E_{p-r, q+r-1}^{r}
$$

Again, the spectral sequence converges to the associated graded $\operatorname{Gr}\left(\mathrm{H}_{\bullet}\right)$, where the homology group $\mathrm{H}_{n}$ has a descending filtration

$$
\mathrm{H}_{n}=F_{(n)}^{n} \supset F_{(n)}^{n-1} \supset F_{(n)}^{n-2} \supset F_{(n)}^{n-3} \supset \ldots
$$

with $F_{(n)}^{p} / F_{(n)}^{p-1}=E_{\infty}^{p, n-p}$.

It is conventional to denote the complexes $E_{r}$ (or $E^{r}$ ) by rectangular arrays, with the index $p$ running in the horizontal direction and the index $q$ running in the vertical direction. So the differential acts down and to the right for a cohomology spectral sequence. It acts up and to the left for a homology spectral sequence.

The Cartan-Leray spectral sequence: For a discrete group, $G$, the Eilenberg-MacLane space, $K(G, n)$ has

$$
\pi_{p}(K(G, n))= \begin{cases}\mathbb{Z} & p=0 \\ G & p=n \\ 0 & \text { otherwise }\end{cases}
$$


(For $n>1, G$ must be abelian.) The group homology of $G$ may be defined as the ordinary homology of the corresponding Eilenberg-MacLane space

$$
\mathrm{H}_{n}(G) \equiv \mathrm{H}_{n}(K(G, 1))
$$

So, we have, immediately that

$$
\mathrm{H}_{0}(G)=\mathbb{Z}, \quad \mathrm{H}_{1}(G)=G /[G, G]
$$

For any abelian discrete group, $G, K(G, 1)$ can be determined from

$$
\begin{aligned}
K(\mathbb{Z}, 1) & =S^{1} \\
K\left(\mathbb{Z}_{n}\right) & =L(\infty, n)=S^{\infty} / \mathbb{Z}_{n} \\
K\left(G \times G^{\prime}, 1\right) & =K(G, 1) \times K\left(G^{\prime}, 1\right)
\end{aligned}
$$

A relatively short computation (see, e.g. [34]) yields

$$
\mathrm{H}_{2}\left(\mathbb{Z}_{m} \times \mathbb{Z}_{n}\right)=\mathbb{Z}_{(m, n)}
$$

If $G$ acts freely on $Y$, the Cartan-Leray spectral sequence allows one to compute the homology of $X=Y / G$. The $E^{2}$ term is

$$
E_{p, q}^{2}=\mathrm{H}_{p}\left(G, \mathcal{H}_{q}(Y)\right)
$$

the homology with twisted coefficients (see [34] for precise definitions). If $G$ acts trivially on $\mathrm{H}_{q}(Y)$, the coefficient group is constant: $\mathcal{H}_{q}(Y)=\mathrm{H}_{q}(Y)$, and the homology groups $\mathrm{H}_{p}\left(G, \mathrm{H}_{q}(Y)\right)$ are determined from $\mathrm{H}_{\bullet}(G)$ and the Universal Coefficients Theorem. If $G$ does not act trivially on $\mathrm{H}_{q}(Y)$, then these homology groups with twisted coefficients are a bit ugly to compute. The one easy case is $\mathrm{H}_{0}\left(G, \mathcal{H}_{q}(Y)\right)$. Let $\mathrm{H}_{q}(Y)_{G}$ be the coinvariant quotient, $\mathrm{H}_{q}(Y)_{G}=\mathrm{H}_{q}(Y) / \mathcal{A}$, where $\mathcal{A}$ is the subgroup of $\mathrm{H}_{q}(Y)$ generated by elements of the form $x-g \cdot x$, for $x \in \mathrm{H}_{q}(Y)$ and $g \in G$. One finds

$$
\mathrm{H}_{0}\left(G, \mathcal{H}_{q}(Y)\right)=\mathrm{H}_{q}(Y)_{G}
$$

If $G$ acts trivially on the homology, then $\mathrm{H}_{q}(Y)_{G}=\mathrm{H}_{q}(Y)$.

The Cartan-Leray spectral sequence converges to $\operatorname{Gr}\left(\mathrm{H}_{\bullet}(Y / G)\right)$. Our basic application will be the following. Assume

- $Y$ is simply connected.

- $G$ acts freely on $Y$. 
- $\mathrm{H}_{2}(Y)_{G}$ is torsion-free.

The $E^{2}$ term of the CLSS looks like.

$$
\begin{array}{cc|ccccc} 
& & \vdots & & & & \\
E_{p, q}^{2} & 2 & \mathrm{H}_{2}(Y)_{G} & & & & \\
& 1 & 0 & 0 & 0 & & \\
& 0 & \mathbb{Z} & \mathrm{H}_{1}(G) & \mathrm{H}_{2}(G) & \mathrm{H}_{3}(G) & \ldots \\
\hline & & 0 & 1 & 2 & 3 &
\end{array}
$$

At least for this portion of the diagram, $d_{2}$ vanishes identically, so $E^{3}=E^{2}$. The differential $d_{3}: \mathrm{H}_{3}(G) \rightarrow \mathrm{H}_{2}(Y)_{G}$ could, in principle, be nontrivial, but vanishes in this case because $\mathrm{H}_{2}(Y)_{G}$ is torsion-free ${ }^{6}$. The spectral sequence converges (since the higher differentials would land outside of this rectangle). So

$$
\mathrm{H}_{1}(X)=\mathrm{H}_{1}(G)=G /[G, G]
$$

and $\mathrm{H}_{2}(X)$ has the filtration

$$
\mathrm{H}_{2}(X)=F_{2} \supset F_{1} \supset F_{0}
$$

where

$$
F_{0}=F_{1}=\mathrm{H}_{2}(Y)_{G}, \quad F_{2} / F_{1}=\mathrm{H}_{2}(G)
$$

This can be rewritten more succinctly as the short exact sequence

$$
0 \rightarrow \mathrm{H}_{2}(Y)_{G} \stackrel{\pi_{*}}{\rightarrow} \mathrm{H}_{2}(X) \rightarrow \mathrm{H}_{2}(G) \rightarrow 0
$$

where $\pi_{*}$ is the push-forward by the projection $\pi: Y \rightarrow X$ and makes sense on the quotient, $\mathrm{H}_{2}(Y)_{G}$, because elements of $\mathcal{A}$ push-forward to zero.

If $\mathrm{H}_{2}(G)=0$, then we find that $\mathrm{H}_{2}(X)$ is just $\mathrm{H}_{2}(Y)_{G}$ and is torsion-free. Otherwise, we still have a nontrivial extension problem, (2.26), to solve and $\mathrm{H}_{2}(X)$ may or may not have torsion.

As a simple application, consider the Tian-Yau manifold, which was the second example in [35]. The K-theory of that manifold has a nontrivial torsion part, but the author of that paper did not compute it. The missing ingredient was a computation of the torsion in $H_{2}(X)$. With our methods, this proves to be straightforward. $\mathrm{H}_{2}(Y)$ is 14-dimensional. Under the action of $G=\mathbb{Z}_{3}$, it decomposes as 4 regular representations and two trivial representations [36]. For each of the regular representations (with basis $x_{1}, x_{2}, x_{3}$,

\footnotetext{
${ }^{6}$ If $\mathrm{H}_{2}(Y)_{G}$ has torsion, then $d_{3}: \mathrm{H}_{3}(G) \rightarrow \mathrm{H}_{2}(Y)_{G}$ kills part of the torsion in $\mathrm{H}_{2}(Y)_{G}$, and the spectral sequence converges at the $E^{4}$ term.
} 
which are cyclically-permuted by $G$ ), we mod out by the subspace spanned by $\left\{x_{1}-x_{2}, x_{2}-x_{3}\right\}$.The quotient is torsion-free, and is generated by $x_{1}$ (modulo $\mathcal{A}$ ). Since $\mathrm{H}_{2}(G)=0$, we conclude that $\mathrm{H}_{2}(X)=\mathrm{H}_{2}(Y)_{G}=\mathbb{Z}^{6}$. The considerations below then yield $K^{0}(X)_{\text {tor }}=K^{1}(X)_{\text {tor }}=\mathbb{Z}_{3}$.

The Atiyah-Hirzebruch spectral sequence: The Atiyah-Hirzebruch spectral sequence allows one to compute the K-theory of $X$, given a knowledge of its cohomology. The $E_{2}$ term is

$$
E_{2}^{p, q}=\mathrm{H}^{p}\left(X, \pi_{q}(B U)\right)
$$

where $\pi_{2 n}(B U)=\mathbb{Z}, \pi_{2 n+1}(B U)=0$. The spectral sequence converges to

$$
E_{\infty}^{p, q}=G r\left(K^{p+q}(X)\right)
$$

The key feature of the differentials $d_{r}$ in the AHSS is their images are always torsion.

We will be interested in the K-theory of Calabi-Yau manifolds, but the computation of the AHSS works exactly the same for any compact, connected 6-manifold, $X$, with finite fundamental group (so that $\mathrm{H}^{1}(X)=0$ ). The $E_{2}$ term looks like

$$
\begin{array}{cc|ccccccc}
6 & 6 & \mathrm{H}^{0}(X) & 0 & \mathrm{H}^{2}(X) & \mathrm{H}^{3}(X) & \frac{\mathrm{H}^{4}(X)}{d_{3}} & \mathrm{H}^{5}(X) & \mathrm{H}^{6}(X) \\
E_{3}^{p, q}=E_{2}^{p, q} & 5 & & & & & & & \\
d_{3} & 4 & \mathrm{H}^{0}(X) & 0 & \mathrm{H}^{2}(X) & \mathrm{H}^{3}(X) & \mathrm{H}^{4}(X) & \mathrm{H}^{5}(X) & \mathrm{H}^{6}(X) \\
& 3 & 0 & 0 & 0 & 0 & 0 & 0 \\
& 2 & \mathrm{H}^{0}(X) & 0 & \mathrm{H}^{2}(X) & \mathrm{H}^{3}(X) & \mathrm{H}^{4}(X) & \mathrm{H}^{5}(X) & \mathrm{H}^{6}(X) \\
& 1 & 0 & 0 & 0 & 0 & 0 & 0 & 0 \\
& 0 & \mathrm{H}^{0}(X) & 0 & \mathrm{H}^{2}(X) & \mathrm{H}^{3}(X) & \mathrm{H}^{4}(X) & \mathrm{H}^{5}(X) & \mathrm{H}^{6}(X) \\
\hline & 0 & 1 & 2 & 3 & 4 & 5 & 6
\end{array}
$$

The first potentially nonvanishing differential is $d_{3}$, since all of the odd rows of the complex vanish. $d_{3}$ annihilates $\mathrm{H}^{0}(X)$, since the "lift" of $\mathrm{H}^{0}(X)$ to $\mathrm{K}$-theory is just the rank, and there is always a trivial vector bundle of rank $n$, for any $n$. So the generator of $\mathrm{H}^{0}(X)$ must survive in the cohomology of $d_{3}$. Similarly, $d_{3}$ annihilates $\mathrm{H}^{2}(X)$, since its "lift" to K-theory is the first Chern class, and for any $\xi \in \mathrm{H}^{2}(X)$, we can always find a line bundle $L$ with $c_{1}(L)=\xi$, so that $L-\mathcal{O}$ is the lift to K-theory of $\xi$. Finally, $d_{3}: \mathrm{H}^{3}(X) \rightarrow \mathrm{H}^{6}(X)$ also vanishes because $\mathrm{H}^{6}(X)$ is torsion-free.

The only possible higher differential, $d_{5}: \mathrm{H}^{0}(X) \rightarrow \mathrm{H}^{5}(X)$ again vanishes for the same reasons as in the previous paragraph, and so the spectral 
sequence converges at the $E_{2}$ term. $K^{0}(X)$ has a filtration by $\mathrm{H}^{e v}(X)$ and $K^{1}(X)$ has a filtration by $\mathrm{H}^{\text {odd }}(X)$.

The filtration on $K^{1}(X)$ leads to the short exact sequence

$$
0 \rightarrow \mathrm{H}^{5}(X) \rightarrow K^{1}(X) \rightarrow \mathrm{H}^{3}(X) \rightarrow 0
$$

Since $\mathrm{H}^{5}(X)$ is torsion, this tells us that the torsion subgroup (which is what we are really interested in computing) is also given by

$$
0 \rightarrow \mathrm{H}^{5}(X) \rightarrow K^{1}(X)_{t o r} \rightarrow \mathrm{H}^{3}(X)_{t o r} \rightarrow 0
$$

The filtration on $K^{0}(X)$ is a little longer. Recasting it as a set of short exact sequences, we have

$$
\begin{gathered}
0 \rightarrow \tilde{K}(X) \rightarrow K^{0}(X) \rightarrow \mathrm{H}^{0}(X) \rightarrow 0 \\
0 \rightarrow \tilde{K}_{(2)}(X) \rightarrow \tilde{K}(X) \rightarrow \mathrm{H}^{2}(X) \rightarrow 0 \\
0 \rightarrow \mathrm{H}^{6}(X) \rightarrow \tilde{K}_{(2)}(X) \rightarrow \mathrm{H}^{4}(X) \rightarrow 0
\end{gathered}
$$

The sequences $(2.30 \mathrm{a}, \mathrm{b})$ are universal. They single out the reduced K-theory, $\tilde{K}(X)$ as the subgroup of $K^{0}(X)$ with vanishing rank and $\tilde{K}_{(2)}(X)$ as the subgroup of the reduced K-theory with vanishing first Chern class. The sequence (2.30c) is special to our low-dimensional situation where the AHSS converges at the $E_{2}$ term.

The image of $\mathrm{H}^{6}(X)$ in $K^{0}(X)$ is $i_{1} \mathcal{O}_{p}$, the push-forward of the trivial line bundle over a point in $X$. This cannot be written as a multiple of some other K-theory class, so $\tilde{K}_{(2)}(X)_{t o r}=\mathrm{H}^{4}(X)_{t o r}$. Putting that together with $(2.30 \mathrm{~b})$, we find that the torsion subgroup is given by the extension

$$
0 \rightarrow \mathrm{H}^{4}(X)_{\text {tor }} \rightarrow K^{0}(X)_{\text {tor }} \rightarrow \mathrm{H}^{2}(X)_{\text {tor }} \rightarrow 0
$$

Now, let us put the output of these two spectral sequences together. Let $Y$ be a Calabi-Yau hypersurface (or complete intersection) in a toric variety. By the Lefschetz theorem, $\mathrm{H}_{1}(Y)=0$ and $\mathrm{H}_{2}(Y)$ is torsion-free. By Poincaré duality, this is enough to show that all of $\mathrm{H}_{\bullet}(Y)$ is torsion-free, and hence so is $\mathrm{H}^{\bullet}(Y)$. Therefore $K^{\bullet}(Y)$ is torsion-free.

To obtain something interesting, we mod out by a freely acting finite group to form $X=Y / G$. For our applications, we will assume that $G$ acts holomorphically, and preserves the holomorphic 3 -form so that $X$ is again Calabi-Yau, but for these topological considerations, that doesn't matter. From $(2.24)$, we learn that $\mathrm{H}^{2}(X)_{\text {tor }}$ and $\mathrm{H}^{5}(X)$ are $G /[G, G] . \mathrm{H}^{3}(X)_{\text {tor }}$ and $\mathrm{H}^{4}(X)_{\text {tor }}=\mathrm{H}_{2}(X)_{\text {tor }}$ are determined once we've solved the extension problem (2.26) to find the torsion in $\mathrm{H}_{2}(X)$. Finally, we use (2.31),(2.29) to compute the torsion in the K-theory. 


\subsection{The quantum symmetry and discrete torsion}

In the $\S 2.5$, we considered Calabi-Yau manifolds of the form $X=Y / G$. String theory propagating on $Y / G$ is governed by an orbifold conformal field theory. Since $G$ acts freely on $Y$, there are no massless states in the twisted sectors, just massive string states. Still, the full conformal field theory (like any orbifold theory) has a quantum symmetry group isomorphic to $G /[G, G]$. How this works in the closed string sector is familiar. A little less familiar is how the quantum symmetry group acts on the D-branes of the theory.

Under tensor products, the flat line bundles on $X$ form an abelian group which is isomorphic to $\mathrm{H}^{2}(X)_{t o r}=G /[G, G]$. Identifying this with the quantum symmetry group, it acts on $K^{\bullet}(X)$ by

$$
v \mapsto v \otimes L
$$

for $L$ a flat line bundle. Since the quantum symmetry is a symmetry of this whole family of conformal field theories, we obtain a further constraint on the monodromies discussed in $\S 2.3$ : they must commute with the action of (2.32).

We saw that $\mathrm{H}_{2}(X)_{\text {tor }} \neq 0$, was the condition for having a potentially nontrivial extension problem (2.31),(2.29) relating the K-theory to the cohomology. It is also the condition under which one can turn on a topologicallynontrivial flat B-field, i.e. one with $H \in \mathrm{H}^{3}(X)_{t o r}$. Turning on this discrete torsion modifies the spectrum of the closed string theory and the D-brane charges take values in twisted $\mathrm{K}$-theory, $K_{H}^{\bullet}(X)$, where $H$ is the class in $\mathrm{H}^{3}(X)_{\text {tor }}[8,9]$. The moduli space of the compactification, in this case, consists of disconnected components, labeled by the discrete torsion.

Viewed as an orbifold of the conformal field theory on $Y$, however, the theory on $X=Y / G$ admits discrete torsion whenever $\mathrm{H}_{2}(G)$ is nonzero, even if $\mathrm{H}_{2}(X)_{t o r}$ (and hence $\mathrm{H}^{3}(X)_{t o r}$ ) vanishes (see (2.26)). In that situation, we have a flat B-field, but there is no topological obstruction to continuously turning it off. Hence the theory "with discrete torsion" is continuously connected to the theory "without" discrete torsion - they lie in the same connected component of the moduli space. We will encounter an example of this in $\S 8$. 


\subsection{Our examples}

Perhaps the best-known Calabi-Yau Manifold is the quintic hypersurface, $Y$, in $\mathbb{C} P^{4}$. The Fermat quintic,

$$
z_{1}^{5}+z_{2}^{5}+z_{3}^{5}+z_{4}^{5}+z_{5}^{5}=0
$$

where the $z_{i}$ are the homogeneous coordinates on $\mathbb{P}^{4}$, is invariant under a freely-acting $\mathbb{Z}_{5} \times \mathbb{Z}_{5}$ symmetry generated by

$$
\left(z_{1}, z_{2}, z_{3}, z_{4}, z_{5}\right) \rightarrow\left(z_{1}, \omega z_{2}, \omega^{2} z_{3}, \omega^{3} z_{4}, \omega^{4} z_{5}\right)
$$

where $\omega^{5}=1$ and

$$
\left(z_{1}, z_{2}, z_{3}, z_{4}, z_{5}\right) \rightarrow\left(z_{2}, z_{3}, z_{4}, z_{5}, z_{1}\right)
$$

The quintic, of course, is simply connected and its K-theory is torsion-free. To form a non-simply connected Calabi-Yau, we mod out by (2.33) to form the manifold $X=Y / \mathbb{Z}_{5}$. X will be our first example. It is nice, in that we can construct the corresponding orbifolded Gepner model quite explicitly, and study the boundary states there.

We can go further and mod out by (2.34) to form $W=X / \mathbb{Z}_{5}$. This will be our second example. Several new features will arise.

\section{BCFT Generalities}

Once we move away from the large-radius limit, we have to use the language of conformal field theory to describe the compactification. A framework for studying D-branes in such compactifications is provided by boundary conformal field theory. A conformal field theory on a Riemann surface with a boundary requires specifying boundary conditions. For $\sigma$-models these conditions are given by Dirichlet or Neumann boundary conditions on the $\sigma$-model fields. Consider now a general conformal field theory with a chiral symmetry algebra $\mathcal{A}$. In this case a class of boundary conditions is provided by the automorphisms $\Omega$ of the chiral algebra. These can be used as gluing conditions for the symmetry generators $W$ along the boundary taken to be the real line: $W(z)=(\Omega \bar{W})(\bar{z})$, for $z=\bar{z}$. A generalization of Cardy's formalism provides, in this case, a set of boundary states, see [37, 38] for more details.

In the context of Calabi-Yau compactification, the chiral algebra which has to be preserved along the boundary, is the $\mathcal{N}=2$ world-sheet supersym- 
metry algebra. This algebra has a well-known automorphism, the mirrorautomorphism. Accordingly, there are two types of boundary conditions [39]:

$$
\begin{array}{ll}
T=\bar{T}, & J=-\bar{J}, \quad G^{ \pm}=\bar{G}^{\mp} \quad \text { "A-type" } \\
T=\bar{T}, & J=\bar{J}, \quad G^{ \pm}=\bar{G}^{ \pm} \quad \text { "B-type" }
\end{array}
$$

These boundary conditions guarantee that the boundary theory preserves one copy of an $\mathcal{N}=2$ algebra. For the construction of BPS branes in CFT it is required also that $\mathcal{N}=1$ spacetime supersymmetry is preserved. The spacetime supersymmetry generator is obtained from the spectral flow operator of the worldsheet theory. Bosonizing the $U(1)$ current $J=i \sqrt{c / 3} \partial X$, the spectral flow operator is given by $\exp (i \sqrt{3 / c} \eta X)$, where $\eta$ specifies the number of units of spectral flow. To construct a space-time SUSY generator, one half unit of spectral flow is needed. The obvious boundary conditions on the spectral flow operator compatible with A-type or B-type boundary conditions are:

$$
\begin{aligned}
& e^{i \sqrt{3 / c} \eta X_{L}}=e^{2 \pi i \varphi} e^{-i \sqrt{3 / c} \eta X_{R}} \quad \text { "A-type" } \\
& e^{i \sqrt{3 / c} \eta X_{L}}=e^{2 \pi i v} e^{i \sqrt{3 / c} \eta X_{R}} \quad \text { "B-type" }
\end{aligned}
$$

We see that the A-type or B-type conditions are Dirichlet or Neumann boundary condition on the boson $X$, describing the bosonized $U(1)$. Accordingly, a "position" can be associated with A-type states and a "Wilson line" with B-type states. The phase appearing in the boundary conditions determines which space-time $\mathcal{N}=1$ algebra is preserved. For two or more D-branes, the difference in the phase determines if there is a common preserved supersymmetry $[40,41]$. $\vartheta$ can be interpreted as a "Wilson line" along the bosonized $U(1)$ for the B-type branes. We will see these Wilson lines explicitly in the case of the Gepner model BPS branes. Similarly, the A-type states have positions along the spectral flow direction.

Far out at large volume, A-type BPS boundary states correspond to branes wrapping special Lagrangian submanifolds of the Calabi-Yau, and B-type BPS branes correspond to vector bundles on holomorphic cycles [39]. In $\mathrm{K}$-theory language, A-type branes are classified by $K^{1}$, whereas $\mathrm{B}$-type branes are classified by $K^{0}$.

For non-BPS states, the spectral flow symmetry can be broken by the boundary, since the brane breaks spacetime supersymmetry. Consistency does however require that the worldsheet supersymmetry is preserved, in other words, there will be A-type and B-type non-BPS states. In the examples introduced in the previous section, we expect to find two types of stable but non-BPS branes, one in $K^{0}$, and one in $K^{1}$. In this paper, we will 
give a physical description of these branes as bound states of BPS D-branes, similar to the approach taken in $[3,42,4,43]$ for orbifolds of tori and flat space.

The aim in the following sections is to identify a stack of BPS D-branes, described as boundary states in Gepner models, whose stable ground state is the torsion brane.

\section{Gepner Models and Orbifolds Thereof}

The stringy regime of Calabi-Yau orbifolds is described by orbifolds of Gepner models, whose closed string sector is reviewed in this section. For this, we first recall the Gepner model and its symmetries and then construct the closed string partition function of the orbifold model.

\subsection{Building blocks of Gepner models}

The basic building blocks of the Gepner model are $\mathcal{N}=2$ minimal models. The minimal model $M M_{k}$ has the following coset representation:

$$
M M_{k}=\frac{S U(2)_{k} \times U(1)_{4}}{U(1)_{2 k+4}}
$$

Accordingly, the central charge is $c_{k}=3 k /(k+2)$. The irreducible representations of the theory are labeled by $(l, m, s)$, where $l$ refers to the $S U(2)$, $m$ to the $U(1)_{2 k+4}$ in the numerator and $s$ to the $U(1)_{4}$ in the nominator. These three integers are subject to an additional constraint:

$$
l+m+s \text { even. }
$$

The symmetry group of a minimal model is $\mathbb{Z}_{2 k+4} \times \mathbb{Z}_{2}$ for $k$ even and $\mathbb{Z}_{4 k+8}$ for $k$ odd. It is generated by operators with the following action on primary fields:

$$
\begin{aligned}
& g \Phi_{(l, m, s)}=e^{2 \pi i \frac{q}{k+2}} \Phi_{(l, m, s)} \\
& h \Phi_{(l, m, s)}=e^{2 \pi i \frac{s}{2}} \Phi_{(l, m, s)}
\end{aligned}
$$

Here, $q$ is the $U(1)$ charge of the primary field $\Phi_{(l, m, s)}$,

$$
q=\frac{m}{k+2}-\frac{s}{2}
$$


The corresponding action on the representation labels is generated by the current $(0,1,1)$, which is interpreted as the spectral flow operator (by $1 / 2$ units), and by $(0,0,2)$, which are the representation labels of a worldsheet supersymmetry generator. The action of these currents on a representation is inherited by the fusion rules. Accordingly, they map $(l, m, s)$ to $(l, m+$ $1, s+1)$, and $(l, m, s)$ to $(l, m, s+2)$. Using these currents, the fields of the minimal model can be organized into orbits. The orbits of the spectral flow operator plays a major role in the discussion of the boundary states.

\subsection{Bulk theory}

To use minimal models for string compactification, we first have to form tensor products of $r$ minimal models in such a way that the central charge is equal to 9. This is the right central charge for a CFT description of a Calabi-Yau compactification. In addition, we need to tensor this with an $S O(2)_{1}$ current algebra, describing the uncompactified directions in the light cone gauge. These products do not give consistent string vacua with four dimensional $\mathcal{N}=1$ space time SUSY. But there exists an orbifold of the tensor product which satisfies all requirements of a consistent string background.

At this point, let us introduce some notation. First, we organize the $l$ quantum numbers in a vector

$$
\lambda=\left(l_{1}, \ldots, l_{r}\right),
$$

where $r$ is the number of minimal models. The quantum numbers $s$ and $m$ are written in an $2 r+1$ dimensional vector:

$$
\mu=\left(s_{0} ; m_{1}, \ldots, m_{r} ; s_{1}, \ldots, s_{r}\right)
$$

One can also define an inner product between these vectors:

$$
\mu \cdot \nu=-\frac{\mu_{0} \nu_{0}}{4}+\sum_{i=1}^{r}\left(\frac{\mu_{i} \nu_{i}}{2\left(k_{i}+2\right)}-\frac{\mu_{i+r} \nu_{i+r}}{4}\right)
$$

Next, we introduce the special $(2 r+1)$-dimensional vectors $\beta_{0}$ with all entries equal to 1 , and $\beta_{j}, j=1, \ldots, r$, having zeroes everywhere except for the 1 st and the $(r+1+j)$ th entry which are equal to 2 . These vectors stand for particular elements in the symmetry group of the tensor product of the minimal model.

To implement worldsheet supersymmetry, one has to project on representations $(\lambda, \mu)$ which satisfy $\beta^{(j)} \cdot \mu \in \mathbb{Z}$. To ensure spacetime supersymmetry, 
one projects on odd integer $U(1)$ charge, i.e. on representations $(\lambda, \mu)$ with $2 \beta_{0} \cdot \mu \in 2 \mathbb{Z}+1$.

The Gepner model partition function is the partition function of the tensor product of minimal models, orbifolded by these symmetries:

$$
\frac{(\operatorname{Im} \tau)^{-2}}{2|\eta(q)|^{2}} \sum_{\lambda,(\mu-\bar{\mu}) \in \Lambda}^{\beta}(-1)^{s_{0}} \chi_{\lambda, \mu}(q) \chi_{\lambda, \bar{\mu}}(\bar{q}) .
$$

The symbol $\sum^{\beta}$ denotes a $\beta$ constrained sum: The sum is taken over those $(\lambda, \mu)$ which fulfill the charge quantization conditions $\mu \cdot \beta_{0} \in 2 \mathbb{Z}+1$ and $\beta^{(j)} \cdot \mu \in \mathbb{Z} . \Lambda$ denotes the lattice spanned by $\beta_{0}$ and $\beta^{(j)}$. In the twisted sectors, the right movers are shifted with respect to the left movers by linear combinations of the lattice vectors in $\Lambda$. They are therefore describing winding modes along $\beta_{0}$ and $\beta^{(j)}$.

The symmetries of the Gepner model are given by the subgroup of symmetries of the tensor product theory which preserve worldsheet and space time supersymmetry [44]. These symmetries act as:

$$
g(\gamma) \Phi_{\lambda \mu ; \bar{\lambda} \bar{\mu}}=\exp i \pi\left(\sum_{j=1}^{r} \frac{\gamma_{j}\left(\mu_{j}+\bar{\mu}_{j}\right)}{k_{j}+2}\right) \Phi_{\lambda \mu ; \bar{\lambda} \bar{\mu}}=e^{\pi i \beta_{\gamma} \cdot(\mu+\bar{\mu})} \Phi_{\lambda \mu ; \bar{\lambda} \bar{\mu}}
$$

where the $\gamma_{i}$ in $\gamma=\left(\gamma_{1}, \ldots, \gamma_{r}\right)$ specify the orbifold action in the individual minimal models as in $(4.2) . \beta_{\gamma}$ is the vector $(0 ; 2 \gamma ; 0)$. For consistency with the projections, we require $\beta_{\gamma} \cdot \beta_{0} \in \mathbb{Z}$.

The new partition function is easy to write down [44]: One just has to include a further vector into the lattice $\Lambda$ and to project on elements $\mu$ with $\beta_{\gamma} \cdot(\mu+\bar{\mu}) \in \mathbb{Z}$. In lattice language, there are new winding modes with $\mu-\bar{\mu}=n \beta_{\gamma}$ coming from twisted sectors.

It is a well-known result by Greene and Plesser that if we orbifold by all generators which are compatible with the charge quantization conditions, we get the mirror theory. In the case of the quintic, the Greene-Plesser group is $\mathbb{Z}_{5}^{3}$.

In this paper, we are interested in free orbifolds of a given Gepner model. Geometrically, we consider free orbifolds of hypersurfaces in weighted projected space.

Our main example is a free orbifold of the quintic. The Gepner model for the quintic is given by a tensor product of five copies of the $k=3$ 
minimal model with the appropriate projections. The orbifold action under consideration is given by

$$
\gamma=(0,1,2,3,4) \quad \text { and } \quad \beta_{1}=\beta_{\gamma}=(0 ; 0,2,4,6,8 ; 0, \ldots, 0)
$$

To obtain the mirror of the orbifold applying the Greene-Plesser construction, we have to orbifold by one further $\mathbb{Z}_{5}$ action. This $\mathbb{Z}_{5}$ action has to be compatible with both the GSO projection and the orbifold projection given by (4.7). This generator can be chosen as $\gamma_{2}=(0,1,3,1,0)$. Taking an orbifold by both $\gamma_{1}$ and $\gamma_{2}$ leads to the mirror of the orbifold [23]. This can also be understood in the following way: We first orbifold the quintic by $\mathbb{Z}_{5}^{3}$, the full Greene-Plesser group of the quintic, to obtain the mirror of the quintic. Then, we mod out by a freely-acting $\mathbb{Z}_{5}$, which is the quantum symmetry associated to orbifolding by $\gamma_{1}$. In this way the original orbifold is undone. Starting with the mirror of the quintic and undoing the orbifold by $\gamma_{1}$ is the same as starting with the quintic and orbifolding by $\gamma_{1}$ and $\gamma_{2}$. The full quantum symmetry of the orbifold is given by $\mathbb{Z}_{5} \times \mathbb{Z}_{5}$, whose generators are denoted $g$ and $h$. The action of these symmetries on the D-branes will be discussed in detail in later sections.

\section{BPS Boundary States for Orbifolds of Gepner Models}

The A-type and B-type boundary states for a single minimal model are determined using Cardy's formalism in rational conformal field theory. In particular, in this framework the boundary states are labeled by the same labels as the primary fields, we denote boundary states using capital letters $(L, M, S)$. In the tensor product, the labels $L$ are summarized in a vector $\Lambda$ which specifies the boundary conditions in the WZW part of the minimal models:

$$
\Lambda=\left(L_{1}, \ldots, L_{r}\right)
$$

The $U(1)$ labels are contained in a lattice vector $\Xi$ :

$$
\Xi=\left(S_{0} ; M_{1}, \ldots, M_{r} ; S_{1}, \ldots, S_{r}\right) .
$$

In this way $(\Lambda, \Xi)$ specifies a consistent boundary state in the tensor product theory.

However, as explained for the closed string situation in the previous section, we have to take an orbifold of the tensor product theory, to ensure space-time and world-sheet supersymmetry. The GSO projection consists of 
a projection on integer charges. In geometrical language, one would say that the bosonized $U(1)$ in eq. (3.2) is compactified on a circle.

A-type boundary states have Dirichlet boundary conditions along the circle. It is well-known from the geometrical context how to construct Atype branes on a circle by summing over images of the translation operator [45]. In our context, the translation operator is given by the spectral flow operator by one half unit. More explicitly, the projected A-type boundary state can be written as $[37,46,47]$

$$
|\Lambda, \Xi\rangle_{p r o j}=\mathrm{const} \sum_{\nu, \nu_{i}}(-1)^{\nu}(-1)^{\frac{s_{0}^{2}}{2}}\left|\lambda, \Xi+\nu \beta_{0}+\nu_{1} \beta^{(1)}+\cdots+\nu_{r} \beta^{(r)}\right\rangle
$$

Inequivalent A-type boundary states are given by orbits of the spectral flow operator and the operators $\beta^{(r)}$. As a consequence, the physically inequivalent choices for $S_{i}$ are encoded in the sum $S=\sum S_{i}$, which is defined mod 4. The $\mathbb{Z}_{2}$ operation $S \mapsto S+2$ maps branes to anti-branes. The A-type states transform non-trivially under the symmetry operations (4.6).

$$
g(\gamma)|(\Lambda, \Xi)\rangle_{\text {proj }}=\left|\left(\lambda, \Xi+\beta_{\gamma}\right)\right\rangle_{\text {proj }}
$$

Note, however, that because we took an orbit under the spectral flow the vector $\gamma_{0}=(1,1,1,1,1)$ acts trivially, and consequently the vectors $\gamma$ and $\gamma+\gamma_{0}$ act in the same way on the boundary state.

B-type boundary states have Neumann boundary conditions on $X$. They are therefore wrapping the circle given by the bosonized $U(1)$. In this case, they should be specified by a discrete Wilson line. In analogy with the situation where D-branes are wrapped on a geometric circle (or rather a lattice vector) this Wilson line is given by $2 \beta_{0} \cdot \Xi$. Similar to the A-type branes, the label $S=\sum S_{i}$ distinguishes between branes and anti-branes. The physically inequivalent choices for the $M_{i}$ can then be encoded in the quantity $M=\sum w_{i} M_{i}$, where $w_{i}$ are the weights of the embedding weighted projected space, $w_{i}=d /\left(k_{i}+2\right)$, where $d=\operatorname{lcm}\left\{k_{i}+2\right\}$. Note that this quantity just extracts the $M$ dependent part of the Wilson line $2 \beta_{0} \cdot \Xi$. The B-type boundary states are invariant under application of elements $\beta_{G P}$ of the Greene-Plesser group since per definition

$$
g\left(\gamma_{G P}\right)\left|\left(\Lambda, 2 \beta_{0} \cdot \Xi\right)\right\rangle=\left|\left(\Lambda, 2 \beta_{0} \cdot\left(\Xi+\beta_{G P}\right)\right)\right\rangle=\left|\left(\Lambda, 2 \beta_{0} \cdot \Xi\right)\right\rangle .
$$

This means that of all the generators in (5.2) there remains only one nontrivially acting symmetry operation on the B-type states,

$$
g: M \mapsto M+2 .
$$


Let us now describe boundary states on the orbifold. A set of A-type boundary states can be obtained by simply projecting the boundary states in (5.1) by the additional orbifold operation. In formulas:

$$
|\Lambda, \Xi\rangle_{o r b}=\mathrm{const} \sum_{\nu, \nu^{\prime}, \nu_{i}}(-1)^{\nu}(-1)^{\frac{s_{0}^{2}}{2}}\left|\lambda, \Xi+\nu \beta_{0}+\nu^{\prime} \beta_{\gamma}+\nu_{1} \beta^{(1)}+\cdots+\nu_{r} \beta^{(r)}\right\rangle \text {. }
$$

This set of A-type states is given by orbits of the spectral flow operator and the additional orbifold operator. To obtain the A-type states for the orbifold of the quintic, plug in the explicit generator $\gamma_{1}$ in (4.7).

Let us turn to the B-type states. There are more B-type states on the orbifold than on the original model. The D-branes of the original model split up into several fractional branes. The reason for this is that the closed string orbifold partition function has additional twisted sector states, the lattice $\Lambda$ in (4.5) is enhanced by an additional vector $\beta_{\gamma}$, which can be wrapped. There are two labels needed to distinguish between different B-type branes, the label $M$ and $M^{1}=d \sum \frac{M_{i} \gamma_{i}}{k_{i}+2}$. Explicit formulas for the B-type states are provided in the appendix.

It is instructive to look at the situation in the language of [16]. They argue that B-type boundary states (with $\Lambda=(0, \ldots, 0)$ ) can be thought of as the restriction of the fractional brane states of a $\mathbb{C}^{r} / \mathbb{Z}_{K}$ orbifold describing the Landau Ginzburg phase of the linear $\sigma$-model to the Calabi-Yau hypersurface. In this picture, the B-type states on a $\mathbb{Z}_{N}$ orbifold correspond to fractional branes on $\mathbb{C}^{r} / \mathbb{Z}_{K} \times \mathbb{Z}_{N}$, which are characterized by the irreducible representations of the orbifold group. For $\mathbb{Z}_{N}$ groups, these representation labels are just given by a phase, and this phase can be mapped to the Wilson-line label introduced before.

The states on the orbifold are only invariant under the Greene-Plesser group of the orbifold, which is a subgroup of the Greene-Plesser group of the original model. The quantum symmetry of the orbifold model is bigger than that of the covering space, and the different fractional branes can be related by applying the symmetry generators. For the quintic orbifold, the B-type states are labeled by $M=\sum M_{i}$ and $M^{1}=M_{2}+2 M_{3}+3 M_{4}+4 M_{5}$. They transform non-trivially under the $\mathbb{Z}_{5} \times \mathbb{Z}_{5}$ quantum symmetry:

$$
\begin{aligned}
& g: M \rightarrow M+2, \quad M^{1} \rightarrow M^{1} \\
& h: M \rightarrow M, \quad M^{1} \rightarrow M^{1}+2
\end{aligned}
$$

$g$ and $h$ can be written in matrix form if we arrange the BPS boundary states in a 25-dimensional vector with entries $\left(M, M^{1}\right)$.

$$
((0,0),(2,0),(4,0),(6,0),(8,0),(2,0),(2,2), \ldots(8,8))^{t}
$$


On these vectors, $g$ acts as a $25 \times 25$ matrix, consisting of 5 dimensional shift matrices on the diagonal. On the other hand, $h$ acts as a shift matrix on those $5 \times 5$ blocks.

These symmetry operations specify the transformation properties of the BPS branes (periods about the Gepner point) under the quantum symmetry, and can be compared with the results obtained from K-theory considerations. The generator $g$ corresponds to the additional $\mathbb{Z}_{5}$ symmetry at the Gepner point. Geometrically, its action on branes is given by the monodromy matrix around the Gepner point. The group generated by $h$ is a universal symmetry group and should be compared to the quantum symmetry discussed in $\S 2.6$. In $\S 7.2$ these symmetry operations are worked out for the example at hand.

So far, we only considered orbifold groups generated by a single group element. Of course, it is possible to repeat the same procedure for an additional generator. In particular, the full Greene-Plesser group can be divided out. In the bulk, this yields the theory on the mirror and the procedure outlined above provides us with a method to confirm that mirror symmetry extends to the open string sector $[48,26,49]$. A-type boundary states on the mirror obtained through an orbifold by the Greene-Plesser group are organized in terms of orbits of this group. The resulting open string sector inherits that structure. Therefore, the projected (A-type) partition function coincides with that found in [37] for B-type states on the original manifold [46]. The Wilson line $M$ on the B-type side becomes an orbit label on the A-type side. Conversely, we can also understand A-type states as B-types on the mirror: Taking successive orbifolds, the B-type states split up further into fractional branes and a new label $d \sum \gamma_{i} M_{i} /\left(k_{i}+2\right)$ has to be introduced after each step. After all Greene-Plesser generators are modded out, the states will be orbits of only the spectral flow operator. In this way, A-type boundary states can be obtained as B-type states on the mirror.

This interpretation provides a convenient way to specify the A-type branes in terms of $d \sum \gamma_{i} M_{i} /\left(k_{i}+2\right)$ for all Greene-Plesser operators. For the quintic we will use the following labels for A-type branes: $M=\sum M_{i}$, $M^{1}=M_{2}+2 M_{3}+3 M_{4}+4 M_{5}$ and $M^{2}=M_{2}+3 M_{3}+M_{4}$. These states transform non-trivially under $\mathbb{Z}_{5} \times \mathbb{Z}_{5} \times \mathbb{Z}_{5}$, which is the quantum symmetry of the mirror of the orbifold.

$$
\begin{gathered}
G: M \rightarrow M+2, \quad M^{1} \rightarrow M^{1} \quad M^{2} \rightarrow M_{2} \\
H: M \rightarrow M, \quad M^{1} \rightarrow M^{1}+2 \quad M_{2} \rightarrow M_{2} \\
H^{\prime}: M \rightarrow M, \quad M^{1} \rightarrow M^{1} \quad M_{2} \rightarrow M_{2}+2
\end{gathered}
$$

In $\S 7.2$, this symmetry action will be compared with the results obtained from K-theory. 


\subsection{The intersection form for the orbifold models}

In this section, we will compute the intersection numbers for the BPS states on the orbifold. Since the torsion state we are looking for is supposed to have zero intersection with all branes, this computation gives a first hint on the symmetry properties of the torsion brane.

In boundary conformal field theory, the intersection form of two branes can be computed as the Witten index in the open string sector, $\operatorname{tr}_{R}(-1)^{F}$. To compute the intersection numbers on the orbifold of the quintic $(k=3)^{5}$, we use the formulas and notation of [10], where the expression for the A-type boundary states with $\Lambda=(0,0,0,0,0)$ was given as

$$
I^{A}=\prod_{i=1}^{5}\left(1-g_{i}^{-1}\right) .
$$

Here, the $g_{i}$ are the symmetry operations in (5.2), with $g_{1}=g((1,0,0,0,0)), \ldots, g_{r}=$ $((0,0,0,0,1))$. Because of the GSO projection the generators $g_{i}$ are not independent and we can express one of them, e.g. $g_{5}$, in terms of the other generators, $\left(g_{5}\right)^{-1}=g_{1} g_{2} g_{3} g_{4}$. This leads to the following expression for the intersection form [10] :

$$
I^{A}=\left(1-g_{1}^{4}\right)\left(1-g_{2}^{4}\right)\left(1-g_{3}^{4}\right)\left(1-g_{4}^{4}\right)\left(1-g_{1} g_{2} g_{3} g_{4}\right) .
$$

For the B-type states on the quintic, all the $g_{i}$ get identified, leaving only the non-trivial generator $g$ of (5.3). The result for the intersection matrix is therefore:

$$
I^{B}=\left(1-g^{-1}\right)^{5}
$$

where $g$ is the generator mapping $M \rightarrow M+2$, where $M=\sum M_{i}$.

On the orbifold we are in an intermediate situation, where some of the $g_{i}$ get identified. The intersection forms can be written in terms of the quantum symmetry generators $g, h$ for the B-type states and in terms of $G, H, H^{\prime}$ for the A-type states. Their action on the boundary states is given in (5.5) and (5.6).

Using $g$ and $h$, the intersection form for B-type states on the orbifold is given by

$$
\begin{aligned}
I_{\text {orb }}^{B}= & g^{4}\left(-h^{4}-h^{3}-h^{2}-h-1\right)+g^{3}\left(2 h^{4}+2 h^{3}+2 h^{2}+2 h+2\right) \\
& +g^{2}\left(-2 h^{4}-2 h^{3}-2 h^{2}-2 h-2\right)+g\left(h^{4}+h^{3}+h^{2}+h+1\right)
\end{aligned}
$$

This means that the intersection matrix depends only on $g$, not on $h$. The intersection matrix between two states which differ only in $M^{1}$, not in $M$ is 
zero, and that of two states which differ only in $M$ equals the intersection number on the quintic. For two states differing by a general application of $g^{n} h^{m}$, the intersection number is independent of $m$.

Using the generators in (5.6), the intersection form for the A-type states can be written as

$$
\begin{aligned}
& I_{\text {orb }}^{A}=G^{4}\left(-1-H^{4} H^{\prime 4}-H^{3} H^{\prime 4}-H^{2}-H H^{\prime 2}\right) \\
& +G^{3}\left(H^{4} H^{\prime 4}+H^{3} H^{\prime 4}+H^{2} H^{\prime 3}+H^{2}+H H^{\prime 4}+H^{\prime 4}+H H^{\prime 2}+H^{\prime}+H^{4} H^{\prime}+H^{3} H^{\prime 2}\right) \\
& +G^{2}\left(-H^{2} H^{\prime 3}-H H^{\prime 4}-H^{\prime 4}-H^{4} H^{\prime 3}-H^{\prime}-H^{4} H^{\prime}-H^{3}-H^{3} H^{\prime 2}-H^{2} H^{\prime}-H H^{\prime}\right) \\
& +G\left(H^{4} H^{\prime 3}+H^{3}+H^{2} H^{\prime}+H H^{\prime}+1\right)
\end{aligned}
$$

States, which only differ by an application of $H$, have zero intersection number. The intersection number is determined by the group actions $G$ and $H^{\prime}$.

\section{Non-BPS States on the Gepner Orbifold}

The D-branes we are interested in are non-BPS but stable. Their stability is guaranteed because, though they are not charged under any RR field, they carry a discrete torsion charge. We are going to construct the torsion brane as a bound state of the BPS branes, whose boundary state description has been given in the previous section. The idea is to form a bound state such that all the BPS charges cancel, leaving only a net torsion charge.

Let us first consider the B-type states. Since the intersection form does not depend on $h$, we conclude that the application of $h$ can only shift the torsion charge. Starting from a given brane $|(\Lambda, \Xi)\rangle$, the RR charge can be canceled by adding a full orbit of $g$-images to that brane. However, for these branes there is no obstruction to decay to the vacuum. To be left with a nontrivial torsion charge, we modify the $g$-action on the orbit by suitable powers of $h$. This is possible, since the state $h^{m} g|(\Lambda, \Xi)\rangle$ has the same intersections as $g|(\Lambda, \Xi)\rangle$, but differs from it by an action on $M^{1}$. Summarizing, we add up the following five branes (taking into account the appropriate Chan-Paton indices):

$$
\begin{aligned}
\left|\left(M, M^{1}\right)\right\rangle+h^{n_{1}} g\left|\left(M, M^{1}\right)\right\rangle+h^{n_{2}} g^{2}\left|\left(M, M^{1}\right)\right\rangle \\
+h^{n_{3}} g^{3}\left|\left(M, M^{1}\right)\right\rangle+h^{n_{4}} g^{4}\left|\left(M, M^{1}\right)\right\rangle \\
\quad=\sum_{m} h^{n_{m}} g^{m}\left|\left(M, M^{1}\right)\right\rangle
\end{aligned}
$$


where

$$
\sum n_{m} \neq 0 \bmod 5
$$

Note, that there are tachyons propagating between the individual branes of this stack of branes. Therefore, this unstable stack of branes can decay into a stable single particle state. We argued that this state is the torsion brane we are looking for. This claim will get further support from the Ktheory analysis in the following section, where the K-theory classes of all BPS boundary states and their symmetry properties will be identified. Taking a sum of K-theory classes as in (6.1) will result in a torsion class.

The A-type states can be discussed similarly. From their intersection form it can be concluded that to cancel the RR charge a sum over all powers of $G$ and $H^{\prime}$ has to be taken. To have a remaining torsion charge, $M^{1}$ should not cancel out. This means that we consider the following stack of branes:

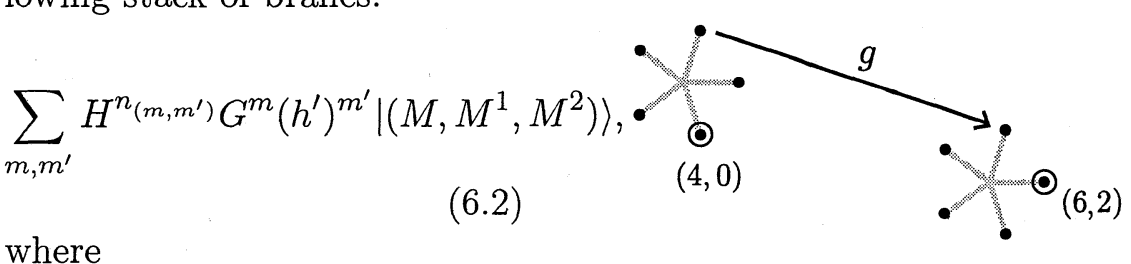

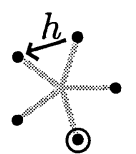

$(8,0)$

where

$$
\sum n_{\left(m, m^{\prime}\right)} \neq 0 \quad \bmod 5
$$

Also for the A-type branes this picture can be verified using $\mathrm{K}$ theory arguments.

To summarize, we have shown that for the free orbifold of the quintic generated by $\gamma_{1}$ there are torsion B-type and A-type states. The A-type states can be reinterpreted as B-type states on the orbifold by $\gamma_{1}$ and $\gamma_{2}$. One might ask what happens if we take an orbifold by the third Greene-Plesser generator. Since this generator does not preserve the projection by $\gamma_{1}$, the torsion states get projected out. This is what is expected from geometry, since the mirror of the quintic has no torsion in K-theory. 


\section{The K-theory of $X$}

\subsection{The K-theory of the Quintic}

First let us review the K-theory of the quintic, $Y$.

The even dimensional cohomology of $Y$ has generators $1, \xi_{2}, \xi_{4}$ and $\xi_{6}$, with relations $\xi_{2}^{2}=5 \xi_{4}, \xi_{2} \cup \xi_{4}=\xi_{6}$ and $\xi_{4}^{2}=0$. The total Chern class of $Y$ is

$$
c(Y)=1+50 \xi_{4}-200 \xi_{6}
$$

$\mathrm{H}^{3}(Y)$ has 204 generators $\zeta_{i}$, and we have $\zeta_{i} \cup \zeta_{j}=\omega_{i j} \xi_{6}$ for some constant antisymmetric matrix $\omega_{i j}$. Also $\xi_{2} \cup \zeta_{i}=\xi_{4} \cup \zeta_{i}=0$.

Let $H$ be the hyperplane line bundle on $Y$, and $D$ be the corresponding hyperplane divisor. Let $C$ be a degree-one rational curve (a $\left.\mathbb{P}^{1}\right)$ in $Y$ and $p$ a point in $Y . K^{0}(Y)$ has the following generators:

\begin{tabular}{|c|c|c|c|c|}
\hline & $r$ & $c_{1}$ & $c_{2}$ & $c_{3}$ \\
\hline \hline $\mathcal{O}$ & 1 & 0 & 0 & 0 \\
$a=i_{!} \mathcal{O}_{D}=H-\mathcal{O}$ & 0 & $\xi_{2}$ & 0 & 0 \\
$b=i_{!} \mathcal{O}_{C}$ & 0 & 0 & $-\xi_{4}$ & $2 \xi_{6}$ \\
$c=i_{!} \mathcal{O}_{p}$ & 0 & 0 & 0 & $2 \xi_{6}$ \\
\hline
\end{tabular}

These correspond simply to a 6-brane wrapped on $Y$, a 4-brane wrapped on $D$, a 2-brane wrapped on $C$ and a 0 -brane sitting at the point $p$.

The relations in the K-theory ring are similar to what we had in cohomology: $a^{2}=5 b, a b=c$ and $b^{2}=0$. For later use, it is also worthwhile knowing that complex conjugation acts by: $\bar{a}=-a+5 b-5 c, \bar{b}=b-2 c$ and $\bar{c}=-c$.

$K^{1}(Y)$ has generators $u_{i} \in \tilde{K}^{0}\left(Y \times S^{1}\right)$ whose only nonvanishing chern class is $c_{2}\left(u_{i}\right)=-\zeta_{i} \cup \phi$, where $\phi$ is the fundamental class of $S^{1}$.

The intersection form on $K^{0}(Y)$ is

$$
(v, w)=\operatorname{Ind}\left(\bar{\partial}_{v \otimes \bar{w}}\right)=\int_{Y} \operatorname{ch}(v \otimes \bar{w}) \operatorname{Td}(Y)
$$

In the above basis, this is the matrix

$$
\Omega=\left(\begin{array}{cccc}
0 & -5 & -1 & -1 \\
5 & 0 & 1 & 0 \\
1 & -1 & 0 & 0 \\
1 & 0 & 0 & 0
\end{array}\right)
$$


The monodromies are generated by the following operations: $M_{r=\infty}: v \mapsto$ $v \otimes H$ at large radius, $M_{c}: v \mapsto v-\operatorname{Ind}\left(\bar{\partial}_{v}\right) \mathcal{O}$ at the conifold and $M_{g}=$ $M_{c} M_{r=\infty}$ at the Gepner point. With respect to the above basis, these are represented by the matrices

$$
\begin{aligned}
M_{r=\infty}= & \left(\begin{array}{llll}
1 & 0 & 0 & 0 \\
1 & 1 & 0 & 0 \\
0 & 5 & 1 & 0 \\
0 & 0 & 1 & 1
\end{array}\right), \quad M_{c}=\left(\begin{array}{cccc}
1 & -5 & -1 & -1 \\
0 & 1 & 0 & 0 \\
0 & 0 & 1 & 0 \\
0 & 0 & 0 & 1
\end{array}\right), \\
M_{g} & =\left(\begin{array}{cccc}
-4 & -10 & -2 & -1 \\
1 & 1 & 0 & 0 \\
0 & 5 & 1 & 0 \\
0 & 0 & 1 & 1
\end{array}\right)
\end{aligned}
$$

Note that $M_{g}^{5}=1$.

To make the connection to CFT at the Gepner point, we need to find a set of five K-theory elements which are cyclically permuted under the $\mathbb{Z}_{5}$ action of $M_{g}$. Set

$$
V_{n}=M_{g}^{-n} \mathcal{O}
$$

or, explicitly,

$$
\begin{aligned}
& V_{1}=\mathcal{O}-a+5 b-5 c \\
& V_{2}=-4 \mathcal{O}+3 a-10 b+5 c \\
& V_{3}=6 \mathcal{O}-3 a+5 b \\
& V_{4}=-4 \mathcal{O}+a \\
& V_{5}=\mathcal{O}
\end{aligned}
$$

Together, these span an index-25 sublattice of $K^{0}(Y)$ - only 2-brane and 0brane charges which are multiples of 5 are realizable as linear combinations of the $V_{n}$. The intersection pairing between these generators is given by the $5 \times 5$ matrix

$$
\left(\begin{array}{ccccc}
0 & 5 & -10 & 10 & -5 \\
-5 & 0 & 5 & -10 & 10 \\
10 & -5 & 0 & 5 & -10 \\
-10 & 10 & -5 & 0 & 5 \\
5 & -10 & 10 & -5 & 0
\end{array}\right)
$$

which agrees with the intersection form (5.8) for the B-type boundary states at the Gepner point. We therefore identify the B-type boundary states constructed in the CFT as BPS representatives of the above K-theory classes.

In fact, as noted by Diaconescu and Douglas [16], the sublattice of $K^{0}(Y)$ which arises in this way is precisely the pullback of the K-theory of $\mathbb{C} P^{4}$ 
under the embedding $Y \hookrightarrow \mathbb{C} P^{4}$. Their rationale for this was that, for the purpose of studying the B-type states, one can ignore the superpotential in the $\mathrm{N}=2$ gauged linear $\sigma$-model.

If one ignores the superpotential, then in the large-radius phase of the GL $\sigma \mathrm{M}$, one has a sigma model with target space $\tilde{Y}=\mathcal{O}_{\mathbb{P}^{4}}(-5)$, the total space of the canonical bundle of $\mathbb{P}^{4}$. This is a noncompact Calabi-Yau manifold, and its $\mathrm{K}$-theory, $K\left(\mathcal{O}_{\mathbb{P}^{4}}(-5)\right)=K\left(\mathbb{P}^{4}\right)$.

\subsection{The K-theory of $X$}

Now we turn to the same computation on $X=Y / \mathbb{Z}_{5}$, where we mod out by (2.33). The cohomology ring of $X$ is a little simpler than for the quintic. $\mathrm{H}^{e v}(X)$ is generated by $1, \xi_{2}^{\prime}, \chi, \xi_{2}^{\prime 2}$, and $\xi_{2}^{\prime 3}$, with relations

$$
\chi \cup \chi=5 \chi=\chi \cup \xi_{2}^{\prime}=\xi_{2}^{\prime 4}=0
$$

The total Chern class of $X$ is

$$
c(X)=1+10 \xi_{2}^{\prime 2}-40 \xi_{2}^{\prime 3}
$$

Under the projection $\pi_{1}: Y \rightarrow X$, we have $\pi_{1}^{*}\left(\xi_{2}^{\prime}\right)=\xi_{2}$, and, consequently, $\pi_{1}^{*}(c(X))=c(Y)$.

$\mathrm{H}^{3}(X)$ has generators $\zeta_{i}, i=1, \ldots 44$, and $\mathrm{H}^{5}(X)$ has a single generator $\chi \cup \zeta$ (the cup product being independent of $i$ ).

Now we turn to the $K$-theory. As before let $H$ be the hyperplane line bundle and let $L$ be the flat line bundle

$$
L=(Y \times \mathbb{C}) / \mathbb{Z}_{5}
$$

where $\mathbb{Z}_{5}$ act by multiplication by a fifth root of unity on $\mathbb{C}$. A basis for $K^{0}(X)$ is

\begin{tabular}{|c|c|c|c|c|}
\hline & $r$ & $c_{1}$ & $c_{2}$ & $c_{3}$ \\
\hline \hline $\mathcal{O}$ & 1 & 0 & 0 & 0 \\
$a=H-\mathcal{O}$ & 0 & $\xi_{2}$ & 0 & 0 \\
$\alpha=L-\mathcal{O}$ & 0 & $\chi$ & 0 & 0 \\
$a^{2}$ & 0 & 0 & $-\xi_{2}^{2}$ & $2 \xi_{2}^{3}$ \\
$a^{3}$ & 0 & 0 & 0 & $2 \xi_{2}^{3}$ \\
\hline
\end{tabular}

where $a \otimes \alpha=0$. Complex conjugation acts by $\bar{a}=-a+a^{2}-a^{3}$ and $\bar{\alpha}=-\alpha$. 
Note that there is no preferred choice for $a$. Shifting $a \rightarrow a^{\prime}=a+\alpha$ yields exactly the same ring. For flat line bundles, there is a preferred one which is actually trivial. There is no similar preferred choice if the line bundle isn't flat.

$K^{1}(X) \subset \tilde{K}^{0}\left(X \times S^{1}\right)$ is given by generators $u_{i}$ whose nonvanishing Chern classes are $c_{2}\left(u_{i}\right)=-\zeta_{i} \cup \phi$ and $\beta$, whose only nonvanishing Chern class is $c_{3}(\beta)=2 \chi \cup \zeta \cup \phi$.

The intersection form on $K^{0}(X)$ is given with respect to the above basis (omitting $\alpha$, which has zero intersection with everything) by

$$
\Omega=\left(\begin{array}{cccc}
0 & -1 & -1 & -1 \\
1 & 0 & 1 & 0 \\
1 & -1 & 0 & 0 \\
1 & 0 & 0 & 0
\end{array}\right)
$$

and the monodromies are generated by the following operations: $M_{r=\infty}$ : $v \mapsto v \otimes H$ at large radius, $M_{c}: v \mapsto v-5 \operatorname{Ind}\left(\bar{\partial}_{v}\right) \mathcal{O}$ at the conifold and $M_{g}=M_{c} M_{r=\infty}$ at the Gepner point.

The conifold monodromy is easy to understand by the considerations of $\S 2.4$. At the conifold point, 5 mutually local particles become massless. They are the wrapped D6-brane and the D6-brane with $p$ torsion D4-branes on it, for $p=1,2,3,4$. These lie in the K-theory classes $\mathcal{O}, L, L^{2}, L^{3}, L^{4}$. By (2.16), the monodromy is

$$
\begin{aligned}
v \mapsto v & -(v, \mathcal{O}) \mathcal{O}-(v, L) L-\left(v, L^{2}\right) L^{2}-\left(v, L^{3}\right) L^{3}-\left(v, L^{4}\right) L^{4} \\
& =v-(v, \mathcal{O})\left(\mathcal{O}+L+L^{2}+L^{3}+L^{3}+L^{4}\right) \\
& =v-5(v, \mathcal{O}) \mathcal{O}
\end{aligned}
$$

where we used the fact that the intersection pairing with $L^{p}$ is independent of $p$ and $\mathcal{O}+L+L^{2}+L^{3}+L^{4}=5 \mathcal{O}$. So, for the purposes of the considerations of $\S 2.4$, we just have five particles carrying 6-brane charge becoming massless at the conifold (instead of one such particle for the quintic).

With respect to the above basis, the above monodromies are represented 
by the matrices

$$
\begin{gathered}
M_{r=\infty}=\left(\begin{array}{llll}
1 & 0 & 0 & 0 \\
1 & 1 & 0 & 0 \\
0 & 1 & 1 & 0 \\
0 & 0 & 1 & 1
\end{array}\right), \quad M_{c}=\left(\begin{array}{cccc}
1 & -5 & -5 & -5 \\
0 & 1 & 0 & 0 \\
0 & 0 & 1 & 0 \\
0 & 0 & 0 & 1
\end{array}\right) \\
M_{g}=\left(\begin{array}{cccc}
-4 & -10 & -10 & -5 \\
1 & 1 & 0 & 0 \\
0 & 1 & 1 & 0 \\
0 & 0 & 1 & 1
\end{array}\right)
\end{gathered}
$$

Another way to think about $\beta$ is as the push-forward $i_{!} \mathcal{O}_{\ell}$ of the trivial line bundle on the torsion 1-cycle, $\ell$. In that case, the torsion pairing between $\alpha$ and $\beta$ consists of computing the holonomy of $L=\mathcal{O}+\alpha$ restricted to $\ell$.

At a generic point in the moduli space, the quantum symmetry is $\mathbb{Z}_{5}$, generated by $v \mapsto v \otimes L$, i.e. $\mathcal{O} \rightarrow \mathcal{O}+\alpha$ and $u_{i} \mapsto u_{i}+\beta$, with all other basis vectors held fixed. Note that the monodromies (in particular, the conifold monodromy) commute with this action. At the Gepner point, the quantum symmetry is enhanced to $\mathbb{Z}_{5} \times \mathbb{Z}_{5}$, where the second $\mathbb{Z}_{5}$ is represented by $M_{g}$ above acting on $K^{0}(X)$, and acts trivially on $K^{1}(X)$. These symmetries preserve both the intersection pairings on $K^{0}(X)$ and $K^{1}(X)$ and the torsion pairing: $K^{0}(X)_{\text {tor }} \times K^{1}(X)_{\text {tor }} \rightarrow \mathbb{Z} / 5 \mathbb{Z}$.

To make contact with the B-type states in the conformal field theory, we follow the procedure above. We write down the orbit of $\mathcal{O}$ under the $\mathbb{Z}_{5} \times \mathbb{Z}_{5}$ quantum symmetry. The orbit generated by $M_{g}$ is

$$
\begin{aligned}
& V_{1,5}=\mathcal{O}-a+a^{2}-a^{3} \\
& V_{2,5}=-4 \mathcal{O}+3 a-2 a^{2}+a^{3} \\
& V_{3,5}=6 \mathcal{O}-3 a+a^{2} \\
& V_{4,5}=-4 \mathcal{O}+a \\
& V_{5,5}=\mathcal{O}
\end{aligned}
$$

The generator of the other $\mathbb{Z}_{5}$ shifts $\mathcal{O} \rightarrow \mathcal{O}+\alpha$. Noting that $6 \alpha=-4 \alpha=\alpha$, this means that it shifts $V_{n, 5} \rightarrow V_{n, 5}+\alpha$. So full $\mathbb{Z}_{5} \times \mathbb{Z}_{5}$ orbit consists of 25 K-theory classes

$$
V_{n, m}=V_{n, 5}-m \alpha
$$

Their intersection form is clearly independent of $m$, and the $n$-dependence 
is given by the $5 \times 5$ matrix

$$
\left(\begin{array}{ccccc}
0 & 1 & -2 & 2 & -1 \\
-1 & 0 & 1 & -2 & 2 \\
2 & -1 & 0 & 1 & -2 \\
-2 & 2 & -1 & 0 & 1 \\
1 & -2 & 2 & -1 & 0
\end{array}\right)
$$

in agreement with the CFT result (5.9). So we identify the 25 B-type boundary states as BPS representatives of the $25 \mathrm{~K}$-theory classes $V_{n, m}$.

Having made this identification, we can give a further, a posteriori argument for the correctness of (7.7), in particular, for our assertion that $M_{c}$ acts trivially on the torsion subgroup. Using this identification, the results of $\S 5$ show that $M_{g}$ acts trivially on the torsion subgroup. We already had that $M_{r=\infty}$ acts trivially on the torsion subgroup, so we conclude that $M_{c}$ acts trivially on the torsion subgroup, as we determined above.

To form a torsion brane representing the class $\alpha$, we sum

$$
V_{1, n_{1}}+V_{2, n_{2}}+V_{3, n_{3}}+V_{4, n_{4}}+V_{5, n_{5}}
$$

with $\sum n_{i}=1 \bmod 5$. This cancels the BPS charges, leaving just the torsion charge. After tachyon condensation, what remains is a non-BPS brane carrying torsion charge $\alpha$.

We have constructed torsion D-branes at large radius and at the Gepner point. This likely means that there is a region of the moduli space, containing both large-radius and the Gepner point, in which the torsion branes are stable. It is not clear that they are stable everywhere. Indeed, in a related situation, Gopakumar and Vafa [50] have argued that the torsion branes might be unstable near the conifold point.

The situation is this: at the conifold, the D6-brane (with or without torsion charge) is becoming massless. If the mass of the "pure" torsion brane stays finite at conifold point, then it clearly becomes unstable to decaying into a D6-brane,anti-D6-brane pair (carrying net torsion charge). If this is the case, then there is a curve of marginal stability surrounding the conifold point, across which the torsion 4-brane becomes unstable.

Of course, the above scenario depends on the behaviour of the mass of the torsion brane as we approach the conifold. Since we don't really know how it behaves, we cannot make a definitive prediction. 


\section{The Permutation Orbifold}

Now we consider the Calabi Yau manifold $W=X / \mathbb{Z}_{5}$, where we mod out by the freely acting $\mathbb{Z}_{5},(2.34)$.

\subsection{Action of the permutation group}

In the Gepner model description of $W$ there is an additional $\mathbb{Z}_{5}$ action, which has to be implemented on the boundary states. The action of that $\mathbb{Z}_{5}$ on the primary fields is given by:

$$
\begin{aligned}
& \sigma:\left(l_{1}, l_{2}, l_{3}, l_{4}, l_{5}\right) \mapsto\left(l_{5}, l_{1}, l_{2}, l_{3}, l_{4}\right), \\
& \sigma:\left(s_{0} ; m_{1}, m_{2}, m_{3}, m_{4}, m_{5} ; s_{1}, s_{2}, s_{3}, s_{4}, s_{5}\right) \mapsto \\
&\left(s_{0} ; m_{5}, m_{1}, m_{2}, m_{3}, m_{4} ; s_{5}, s_{1}, s_{2}, s_{3}, s_{4}\right) .
\end{aligned}
$$

It is easy to see how this action translates into an action on boundary states in the tensor product theory:

$$
\begin{aligned}
& \Lambda=\left(L_{1}, L_{2}, L_{3}, L_{4}, L_{5}\right) \mapsto\left(L_{5}, L_{1}, L_{2}, L_{3}, L_{4}\right) \\
&\left(S_{0} ; M_{1}, M_{2}, M_{3}, M_{4}, M_{5} ; S_{1}, S_{2}, S_{3}, S_{4}, S_{5}\right) \mapsto \\
&\left(S_{0} ; M_{5}, M_{1}, M_{2}, M_{3}, M_{4} ; S_{5}, S_{1}, S_{2}, S_{3}, S_{4}\right)
\end{aligned}
$$

In the following, it is assumed that the boundary states under consideration carry the same $L$ labels in all minimal models $\Lambda=(L, L, L, L, L)$. In particular, we are interested in the B-type boundary states with $\Lambda=(0,0,0,0,0)$. As a first step, let us consider an action of the permutation group in the unorbifolded $(k=3)^{5}$ theory. The B-type branes, being labeled by $M=\sum M_{i}$, are invariant under the action of the permutation group. This is no longer the case on the orbifold $X$, since $\gamma$ acts differently on the individual minimal model factors. The permutation acts on the branes on the orbifold as:

$$
\left|(0,0,0,0,0), M, M^{1}\right\rangle \mapsto\left|(0,0,0,0,0), M,\left(M^{1}+M\right)\right\rangle
$$

As a consequence, the five states with $M=0$ are invariant under the cyclic permutation. The other 20 states can be organized in four $\mathbb{Z}_{5}$ orbits of length five. Note that the action of the permutation symmetry is the same as that of an ( $M$ dependent) power of $h$, meaning that the four orbits are formed in such a way that the resulting brane configuration can be lifted to the quintic. In particular, each orbit is labeled by $M$. 
To study the branes on the orbifold $\mathrm{W}$ it is important to realize that the generator $\sigma$ of the cyclic permutation commutes with the generator $g\left(\gamma_{1}\right)$ only up to a generator of the GSO projection $g_{0}$. This reflects the fact that geometrically these two actions only commute up to projective equivalence of the embedding projective space. The full orbifold group is therefore a non-abelian group $\Gamma$ with the relation:

$$
g\left(\gamma_{1}\right) \sigma=g_{0} \sigma g\left(\gamma_{1}\right)
$$

The commutator subgroup is just the $\mathbb{Z}_{5}$ subgroup generated by $g_{0}$, and we have the exact sequence of groups

$$
0 \rightarrow \mathbb{Z}_{5} \rightarrow \Gamma \rightarrow \mathbb{Z}_{5} \times \mathbb{Z}_{5} \rightarrow 0
$$

The quantum symmetry group of the orbifold model is $\Gamma /[\Gamma, \Gamma]=\mathbb{Z}_{5} \times \mathbb{Z}_{5}$.

To classify the branes, we have to find all irreducible representation of $\Gamma$. Each representation can be used as an action on the Chan-Paton factors and therefore specifies a brane. We immediately see that the orbits of four which we found above correspond to irreducible representations of the orbifold group, where $g_{0}$ acts as a phase multiplication:

$$
g\left(\gamma_{1}\right) \sigma=e^{\frac{\pi i}{5} M} \sigma g\left(\gamma_{1}\right) \quad M=0,2,4,6,8
$$

In addition to this we find 25 one dimensional representations, where $g_{0}$ acts trivially. In that case, $g\left(\gamma_{1}\right)$ and $\sigma$ generate an abelian group. To check if this covers all irreducible inequivalent representations, we apply a lemma of Burnside, which states that the sum of the square of the dimensions of the irreducible representations equals the order of the group. Since the order of the group is $5^{3}$, these 29 representations are indeed all irreducible inequivalent representations. The representations of the full non-abelian group can be reinterpreted in terms of representations of the group generated by $g\left(\gamma_{1}\right)$ and $\sigma$. This is the group which has a direct interpretation in geometry. We see that for $M=0$ this group acts in one-dimensional representations, and we have the usual picture of fractional branes labeled by two phases. For $M \neq 0$ this groups acts in a projective representation on the Chan-Paton factors. In other words, we have turned on discrete torsion. The two-cocycle in $H^{2}(\Gamma, U(1))$ is specified by the phase factor appearing in the projective representation:

$$
\left.\epsilon\left(g\left(\gamma_{1}\right)^{m} \sigma^{n}, g\left(\gamma_{1}\right)^{p} \sigma^{q}\right)\right)=e^{\frac{\pi i}{5} M(m q-p n)}
$$

The quantum symmetry of the model, $\Gamma /[\Gamma, \Gamma]=\mathbb{Z}_{5} \times \mathbb{Z}_{5}$, is the same quantum symmetry group that we see at a generic point in the moduli space. In contrast to the previous cases, there is no enhanced symmetry at the Gepner point. 


\subsection{The K-theory of $W$}

$\mathrm{H}^{e v}(W)$ is generated by $1, \xi_{2}^{\prime \prime}, \chi_{1}, \chi_{2}, \xi_{4}^{\prime \prime}$ and $\xi_{6}^{\prime \prime}$, with relations

$$
\xi_{2}^{\prime \prime 2}=25 \xi_{4}^{\prime \prime}, \quad \xi_{2}^{\prime \prime} \cup \xi_{4}^{\prime \prime}=\xi_{6}^{\prime \prime}, \quad \xi_{2}^{\prime \prime} \cup \chi_{i}=\xi_{4}^{\prime \prime} \cup \chi_{i}=5 \chi_{i}=\chi_{i} \cup \chi_{j}=0
$$

The total Chern class of $W$ is

$$
c(W)=1+10 \xi_{4}^{\prime \prime}-8 \xi_{6}^{\prime \prime}
$$

Under the projection $\pi_{2}: X \rightarrow W$,

$$
\pi_{2}^{*}\left(\xi_{2}^{\prime \prime}\right)=5 \xi_{2}^{\prime}, \quad \pi_{2}^{*}\left(\xi_{4}^{\prime \prime}\right)=\xi_{2}^{\prime 2}, \quad \pi_{2}^{*}\left(\chi_{1}\right)=\chi, \quad \pi_{2}^{*}\left(\chi_{2}\right)=0
$$

and consequently $\pi_{2}^{*}(c(W))=c(X)$.

Note that the generator of $\mathrm{H}^{2}(W)$ pulls back to 5 times the generator of $\mathrm{H}^{2}(X)$. Equivalently, the generator of $\mathrm{H}_{2}(X)$ pushes forward to 5 times the generator of $\mathrm{H}_{2}(W)$. Recall that the Cartan-Leray spectral sequence tells us that $\pi_{1 *}: \mathrm{H}_{2}(Y) \rightarrow \mathrm{H}_{2}(X)$ is an isomorphism, since $\mathrm{H}_{2}\left(\mathbb{Z}_{5}\right)=0$. On the other hand, applying the Cartan-Leray spectral sequence to $W=$ $Y /\left(\mathbb{Z}_{5} \times \mathbb{Z}_{5}\right)$, one has an extension

$$
0 \rightarrow \mathrm{H}_{2}(Y) \stackrel{\pi_{*}}{\rightarrow} \mathrm{H}_{2}(X) \rightarrow \mathbb{Z}_{5} \rightarrow 0
$$

since $\mathrm{H}_{2}\left(\mathbb{Z}_{5} \times \mathbb{Z}_{5}\right)=\mathbb{Z}_{5}$. There are two possible extensions: either the sequence splits, or it does not. In fact, Aspinwall and Morrison [23] show that the generator of $\mathrm{H}_{2}(W)$ is represented by an elliptic curve, $E$, which is not the image of an element in $\mathrm{H}_{2}(Y)$. Hence the sequence does not split, $\pi_{*}=\pi_{2 *} \circ \pi_{1 *}$ is multiplication by 5 and therefore so is $\pi_{2 *}$.

Note that we are in the situation discussed in $\S 2.6$, where $\mathrm{H}^{3}(W)$ is torsion-free, whereas $\mathrm{H}_{2}(G) \neq 0$. Viewed as an orbifold CFT, the model on $W$ admits discrete torsion, but this can be continuously turned off. As a consequence, the Kähler moduli space of $W$ is a fivefold cover of the moduli space of $X$, branched at the Gepner point and the large-radius point. As we saw in $\S 8.1$, there is no enhancement of the quantum symmetry at the Gepner point and so the Gepner point is now a smooth point in the moduli space. Since we have a fivefold cover, the coordinate on the moduli space is now $\psi$ rather than $\psi^{5}$. Points related by multiplying $\psi$ by a fifth root of unity are related by adding one unit of discrete torsion (8.7). Hence they are physically inequivalent, as was already noted by [23].

A basis for $K^{0}(W)$ is 


\begin{tabular}{|c|c|c|c|c|}
\hline & $r$ & $c_{1}$ & $c_{2}$ & $c_{3}$ \\
\hline \hline $\mathcal{O}$ & 1 & 0 & 0 & 0 \\
$a=S-\mathcal{O}$ & 0 & $\xi_{2}^{\prime \prime}$ & 0 & 0 \\
$\alpha_{1}=L_{1}-\mathcal{O}$ & 0 & $\chi_{1}$ & 0 & 0 \\
$\alpha_{2}=L_{2}-\mathcal{O}$ & 0 & $\chi_{2}$ & 0 & 0 \\
$b=i_{!} \mathcal{O}_{E}$ & 0 & 0 & $-\xi_{4}^{\prime \prime}$ & 0 \\
$c=i_{!} \mathcal{O}_{p}$ & 0 & 0 & 0 & $2 \xi_{6}^{\prime \prime}$ \\
\hline
\end{tabular}

The line bundle $S$ on $W$ satisfies $\pi_{2}^{*} S=H^{5}$. the ring structure is $a^{2}=$ $25(b+c), a b=c$ and $a \alpha_{i}=b \alpha_{i}=0$.

The intersection form, in the above basis (omitting the $\alpha_{i}$ ), is

$$
\Omega=\left(\begin{array}{cccc}
0 & -5 & 0 & -1 \\
5 & 0 & 1 & 0 \\
0 & -1 & 0 & 0 \\
1 & 0 & 0 & 0
\end{array}\right)
$$

The monodromy about the large radius is generated by $M_{r=\infty}: v \mapsto v \otimes S$ or, in the above basis,

$$
M_{r=\infty}=\left(\begin{array}{cccc}
1 & 0 & 0 & 0 \\
1 & 1 & 0 & 0 \\
0 & 25 & 1 & 0 \\
0 & 25 & 1 & 1
\end{array}\right)
$$

There are 5 conifold points, and the monodromies about them are generated by

$$
\begin{aligned}
& M_{c_{i}}: v \mapsto v-\left(v, w_{i}\right) w_{i}, \quad i=1, \ldots 4 \\
& M_{c_{5}}: v \mapsto v-25(v, \mathcal{O}) \mathcal{O}
\end{aligned}
$$

where

$$
\begin{aligned}
& w_{1}=5 \mathcal{O}-4 a+90 b+6 c \\
& w_{2}=5 \mathcal{O}-3 a+60 b+8 c \\
& w_{3}=5 \mathcal{O}-2 a+35 b+7 c \\
& w_{4}=5 \mathcal{O}-a+15 b+4 c
\end{aligned}
$$

These classes (including $k=5$, if one formally writes $w_{5}=5 \mathcal{O}$ ) can be written more succinctly as

$$
w_{k}=5 \mathcal{O}+(k-5) a+\frac{5}{2}(k-5)(k-10) b+\frac{1}{6} k(k-5)(k-10) c
$$


On the mirror of $W$, four of the five conifold points correspond to singularities at which an $S^{3}$ shrinks to zero size. At the mirror of the fifth, there are five singularities, each of which locally looks like $S^{3} / \mathbb{Z}_{5}$. D-branes in such backgrounds were studied in [50], with the result that five states should become massless when the $S^{3} / \mathbb{Z}_{5}$ shrinks to zero. This result is consistent with the computation of the $F_{1}$-function in [23]. The number of massless hypermultiplets can be read off from the coefficient of the logarithm in an expansion of $F_{1}$ about the singular point. At $\psi=1$, there are 25 massless states. At the other four conifold points, there is only one massless state.

In our case, the multiplicity 25 in $M_{c_{5}}$ is easy to understand: as in (7.7), there are 25 6-branes (flat line bundles on $W$ ) which become massless at $\psi=1$. Near the other four points, the conformal field theory is the same as the theory near $\psi=1$, but with discrete torsion turned on. As we saw in $\S 8.1$, the remaining irreducible representations of the orbifold group on the Chan-Paton factors correspond to rank-5 projective bundles. But, since the discrete torsion is topologically trivial, we can choose an isomorphism between the twisted differential $\mathrm{K}$-theory and the ordinary $\mathrm{K}$-theory of $W$, and write these in terms of ordinary $\mathrm{K}$-theory classes, $w_{i}$ on $W$. The rank of the $w_{i}$ are, of course, 5 . The first Chern class (and hence the coefficient of $a$ ) can be understood as follows. The action of a $2 \pi$ rotation in the $\psi$-plane is to tensor with $S$, which shifts the first Chern class (and hence the coefficient of a) by 5 . A rotation by $2 \pi / 5$ should therefore shift the first Chern class (and hence the coefficient of $a$ ) by 1 . Having understood these two coefficients, the rest are determined by requiring

$$
w_{k+5}=w_{k} \otimes S
$$

and requiring that the coefficients of $a, b$, and $c$ vanish for $k=5$. The unique solution to these constraints is (8.14).

In the above basis,

$$
\begin{aligned}
M_{c_{1}} & =\left(\begin{array}{cccc}
-69 & -575 & -20 & -25 \\
56 & 461 & 16 & 20 \\
-1260 & -10350 & -359 & -450 \\
-84 & -690 & -24 & -29
\end{array}\right), \\
M_{c_{2}} & =\left(\begin{array}{cccc}
-34 & -425 & -15 & -25 \\
21 & 256 & 9 & 15 \\
-420 & -5100 & -179 & -300 \\
-56 & -680 & -24 & -39
\end{array}\right)
\end{aligned}
$$




$$
\begin{gathered}
M_{c_{3}}=\left(\begin{array}{cccc}
-14 & -300 & -10 & -25 \\
6 & 121 & 4 & 10 \\
-105 & -2100 & -69 & -175 \\
-21 & -420 & -14 & -34
\end{array}\right), \quad M_{c_{4}}=\left(\begin{array}{cccc}
-4 & -200 & -5 & -25 \\
1 & 41 & 1 & 5 \\
-15 & -600 & -14 & -75 \\
-4 & -160 & -4 & -19
\end{array}\right), \\
M_{c_{5}}=\left(\begin{array}{cccc}
1 & -125 & 0 & -25 \\
0 & 1 & 0 & 0 \\
0 & 0 & 1 & 0 \\
0 & 0 & 0 & 1
\end{array}\right)
\end{gathered}
$$

Multiplying these monodromies together, we find the monodromy at the Gepner point is

$$
M_{g}=M_{c_{1}} M_{c_{2}} M_{c_{3}} M_{c_{4}} M_{c_{5}} M_{r=\infty}=1
$$

confirming that the Gepner point is a smooth point in the moduli space, as we argued in $\S 8.1$.

In contrast to the previous example, (8.11) and (8.12) are not the unique set of monodromies compatible with all of our criteria. For instance, choose a particular torsion element, $\gamma \in K^{0}(W)_{t o r}$, and modify (8.12a) to read

$$
M_{c_{i}}: v \mapsto v-\left(v, w_{i}\right)\left(w_{i}+\gamma\right), \quad i=1, \ldots, 4
$$

This still acts trivially on the torsion subgroup, it induces the same action on $K^{0}(W) / K^{0}(W)_{\text {tor }}$ as before and, moreover, it still satisfies (8.15) (because $\left.\sum_{i=1}^{4} w_{i}=0 \bmod 5\right)$.

Clearly more physical input is required to completely pin down these monodromies in the general case. That is an interesting subject for future work.

\section{Acknowledgements}

We would like to thank V. Braun, E. Diaconescu, M. Douglas, B. Fiol, G. Moore, T. Pantev, C. Römelsberger and C. Vafa for discussions. J.D. would also like to thank D. Freed for invaluable mathematical advice. Furthermore, we are grateful to the Erwin Schrödinger Institute for hospitality during the workshop "Duality, String Theory and M-Theory," where some of this work was carried out. 


\section{A Explicit Formulas for B-Type States on Gepner Orbifolds.}

In this section, we provide explicit formulas for B-type boundary states on Gepner orbifolds. For concreteness, we include one orbifold generator $\gamma$, and a corresponding lattice vector $\beta_{1}=(0 ; 2 \gamma ; 0,0,0,0,0)$. We assume that $\gamma$ generates a free orbifold group of order $d=l \mathrm{~cm}\left\{k_{i}+2\right\}$. The closed string partition function is (4.5) where the lattice $\Lambda$ is generated by $\beta_{0}, \beta^{(r)}, \beta_{1}$.

B-type boundary states are written as a linear combination of Ishibashi states built on bulk fields, which fulfill B-type boundary conditions. We impose B-type boundary conditions in each minimal model separately, and therefore have to take bulk fields where $\mu=-\bar{\mu}$. In addition, we have to require that the bulk field is contained in the closed string partition function:

$$
\bar{\mu}=\mu+n \beta_{0}+n_{i} \beta^{(i)}+m \beta_{1}=-\mu .
$$

In other words, all B-type Ishibashi states are built on winding modes of the lattice $\Lambda$.

Following Recknagel and Schomerus, a consistent boundary state can then be obtained as

$$
\left.|\alpha\rangle=P \sum_{\lambda \mu}(-1)^{\frac{s_{0}^{2}}{2}} B_{\alpha}^{\lambda \mu}|\lambda \mu\rangle\right\rangle .
$$

Here, $|\lambda \mu\rangle\rangle$ is the Ishibashi state built on the primary field of the representation $(\lambda, \mu), P$ is a projection operator which will be discussed in more detail below and $B_{\alpha}^{\lambda \mu}$ is given by Cardy:

$$
B_{\alpha}^{\lambda, \mu}=\prod_{j=1}^{r} \frac{1}{\sqrt{\sqrt{2}\left(k_{j}+2\right)}} \frac{\sin \left(l_{j}, L_{j}\right)_{k_{j}}}{\sqrt{\sin \left(l_{j}, 0\right)_{k_{j}}}} e^{2 \pi i \mu \cdot \Xi} .
$$

The projector $P$ has to make sure that we sum over all winding modes, and that the integer charge and spin alignment conditions are fulfilled.

$$
\begin{aligned}
P=\sum_{m \in \mathbb{Z}_{d}} \sum_{n \in \mathbb{Z}_{K / 2}} \prod_{i=1}^{r} \frac{1}{k_{i}+2} \sum_{\rho_{i} \in \mathbb{Z}_{k_{i}+2}} e^{\frac{2 \pi i}{k_{i}+2}\left(m_{i}+n+m \gamma_{i}\right) \rho_{i}} \\
\frac{1}{K} \sum_{\nu \in \mathbb{Z}_{K}} e^{\pi i\left(2 \mu \cdot \beta_{0}-1\right) \nu} \prod_{i=1}^{r} \frac{1}{2} \sum_{\nu_{i} \in \mathbb{Z}_{2}} e^{2 \pi i \beta^{(i)} \cdot \mu}
\end{aligned}
$$


The open string partition function computed from these boundary states is given by

$$
\begin{aligned}
Z_{\left(\tilde{\Lambda}, \tilde{M}, \tilde{M}^{1}\right)\left(\Lambda, M, M^{1}\right)}=\mathrm{const} & \sum_{\lambda^{\prime} \mu^{\prime}} \delta_{d \gamma \cdot\left(\tilde{\Xi}-\Xi-\mu^{\prime}\right)}^{(d)} \delta_{\sum \frac{K}{2 k_{i}+4}\left(\tilde{M}_{i}-M_{i}-\nu-m_{i}^{\prime}\right)}^{(K / 2)} \\
& \prod_{i} N_{\tilde{L}_{i} L_{i}}^{l_{i}^{\prime}} \delta_{\nu-M_{i}-\tilde{M}_{i}+m_{i}^{\prime}}^{(2)} \delta_{\nu-2 \nu_{i}+S_{i}-\tilde{S}_{i}+s_{i}^{\prime}}^{(4)}
\end{aligned}
$$

This is almost the same partition function as for B-type states on the quintic, but there is an additional $\delta$-function insertion in the open string sector (the first $\delta$-function in the above equation). In words, this $\delta$-function enforces that all momenta $\mu$ propagating in the open string sector are correctly quantized along the direction $\beta_{1}$. (At this point it is easy to also describe boundary states on orbifolds with more than one generator: The only difference is that there are additional $\delta$-functions imposing the correct momentum quantization in the open string partition function.) The other $\delta$-functions make sure that the correct charge quantization is satisfied. We read off from the partition function, that the boundary conditions are completely determined by $\Lambda, d \gamma \cdot \Xi$ and $\sum M_{i}$, as noted in the text before. The quantities $d \gamma \cdot \Xi$ and $\sum M_{i}$ appear as Wilson lines in this formula: In the open string sector they determine a shift in the momentum quantization along a particular direction. For the spectral flow operator $\beta_{0}$ this shift determines if the two branes preserve the same supersymmetry. In [41] this was related to a grading on the space of branes. Here we see that the additional orbifold induces a similar kind of grading. It would be interesting to interpret this further.

\section{References}

[1] R. Minasian and G. Moore, "K-theory and Ramond-Ramond charge," JHEP 11 (1997) 002, hep-th/9710230.

[2] E. Witten, "D-branes and K-theory," JHEP 12 (1998) 019, hep-th/9810188.

[3] O. Bergman and M. R. Gaberdiel, "Stable non-BPS D-particles," Phys. Lett. B441 (1998) 133-140, hep-th/9806155.

[4] A. Sen, "Non-BPS states and branes in string theory," hep-th/9904207.

[5] D.-E. Diaconescu and J. Gomis, "Fractional branes and boundary states in orbifold theories," JHEP 10 (2000) 001, hep-th/9906242. 
[6] D. S. Freed, "Dirac charge quantization and generalized differential cohomology," hep-th/0011220.

[7] D. S. Freed and M. J. Hopkins, "On Ramond-Ramond fields and K-theory," JHEP 05 (2000) 044, hep-th/0002027.

[8] A. Kapustin, "D-branes in a topologically nontrivial B-field," hep-th/9909089.

[9] P. Bouwknegt and V. Mathai, "D-branes, B-fields and twisted K-theory," JHEP 03 (2000) 007, hep-th/0002023.

[10] I. Brunner, M. R. Douglas, A. Lawrence, and C. Römelsberger, "D-branes on the quintic," JHEP 08 (2000) 015, hep-th/9906200.

[11] D.-E. Diaconescu and C. Romelsberger, "D-branes and bundles on elliptic fibrations," Nucl. Phys. B574 (2000) 245-262, hep-th/9910172.

[12] P. Kaste, W. Lerche, C. A. Lutken, and J. Walcher, "D-branes on K3-fibrations," Nucl. Phys. B582 (2000) 203-215, hep-th/9912147.

[13] E. Scheidegger, "D-branes on some one- and two-parameter Calabi-Yau hypersurfaces," JHEP 04 (2000) 003, hep-th/9912188.

[14] S. Govindarajan and T. Jayaraman, "On the Landau-Ginzburg description of boundary CFTs and special Lagrangian submanifolds," JHEP 07 (2000) 016, hep-th/0003242.

[15] S. Govindarajan, T. Jayaraman, and T. Sarkar, "Worldsheet approaches to D-branes on supersymmetric cycles," Nucl. Phys. B580 (2000) 519-547, hep-th/9907131.

[16] D.-E. Diaconescu and M. R. Douglas, "D-branes on stringy Calabi-Yau manifolds," hep-th/0006224.

[17] P. Mayr, "Phases of supersymmetric D-branes on Kähler manifolds and the McKay correspondence," hep-th/0010223.

[18] A. Tomasiello, "D-branes on Calabi-Yau manifolds and helices," hep-th/0010217.

[19] S. Govindarajan and T. Jayaraman, "D-branes, exceptional sheaves and quivers on Calabi-Yau manifolds: From Mukai to McKay," hep-th/0010196.

[20] M. R. Douglas, B. Fiol, and C. Römelsberger, "Stability and BPS branes," hep-th/0002037. 
[21] M. R. Douglas, B. Fiol, and C. Römelsberger, "The spectrum of BPS branes on a noncompact Calabi-Yau," hep-th/0003263.

[22] M. R. Douglas, "D-branes, categories and $N=1$ supersymmetry," hep-th/0011017.

[23] P. S. Aspinwall and D. R. Morrison, "Chiral rings do not suffice: $N=(2,2)$ theories with nonzero fundamental group," Phys. Lett. B334 (1994) 79-86, hep-th/9406032.

[24] D. S. Freed and E. Witten, "Anomalies in string theory with D-branes," hep-th/9907189.

[25] M. Bershadsky, C. Vafa, and V. Sadov, "D-branes and topological field theories," Nucl. Phys. B463 (1996) 420-434, hep-th/9511222.

[26] M. Kontsevich, "Homological algebra of mirror symmetry," ICM Zurich (1994) alg-geom/9411018.

[27] R. P. Horja, "Hypergeometric functions and mirror symmetry in toric varieties," math.AG/9912109.

[28] B. R. Greene and Y. Kanter, "Small volumes in compactified string theory," Nucl. Phys. B497 (1997) 127-145, hep-th/9612181.

[29] M. Atiyah, V. Patodi, and I. Singer, "Spectral asymmetry and Riemannian geometry. I," Math. Proc. Camb. Phil. Soc. 77 (1975) 43.

[30] M. Atiyah, V. Patodi, and I. Singer, "Spectral asymmetry and Riemannian geometry. II," Math. Proc. Camb. Phil. Soc. 78 (1975) 405 .

[31] M. Atiyah, V. Patodi, and I. Singer, "Spectral asymmetry and Riemannian geometry. III," Math. Proc. Camb. Phil. Soc. 79 (1976) 71.

[32] I. Brunner and J. Distler. Work in progress.

[33] R. Bott and L. Tu, Differential Forms in Algebraic Topology. Springer-Verlag, 1982.

[34] K. Brown, Cohomology of Groups. Springer-Verlag, 1982.

[35] V. Braun, "K-theory torsion," hep-th/0005103.

[36] J. Distler, B. Greene, K. Kirklin, and P. Miron, "Evaluation of $\overline{27}^{3}$ Yukawa couplings in a three generation superstring model," Phys. Lett. 195B (1987) 41. 
[37] A. Recknagel and V. Schomerus, "D-branes in Gepner models," Nucl. Phys. B531 (1998) 185-225, hep-th/9712186.

[38] J. L. Cardy, "Boundary conditions, fusion rules and the Verlinde formula," Nucl. Phys. B324 (1989) 581.

[39] H. Ooguri, Y. Oz, and Z. Yin, "D-branes on Calabi-Yau spaces and their mirrors," Nucl. Phys. B477 (1996) 407-430, hep-th/9606112.

[40] M. Gutperle and Y. Satoh, "D-branes in Gepner models and supersymmetry," Nucl. Phys. B543 (1999) 73-104, hep-th/9808080.

[41] M. R. Douglas, "D-branes, categories and $N=1$ supersymmetry," hep-th/0011017.

[42] M. R. Gaberdiel and J. Bogdan Stefanski, "Dirichlet branes on orbifolds," Nucl. Phys. B578 (2000) 58-84, hep-th/9910109.

[43] M. R. Gaberdiel, "Lectures on non-BPS Dirichlet branes," Class. Quant. Grav. 17 (2000) 3483-3520, hep-th/0005029.

[44] B. R. Greene and M. R. Plesser, "Duality in Calabi-Yau moduli space," Nucl. Phys. B338 (1990) 15-37.

[45] W. Taylor, "D-brane field theory on compact spaces," Phys. Lett. B394 (1997) 283-287, hep-th/9611042.

[46] I. Brunner and V. Schomerus, "D-branes at singular curves of Calabi-Yau compactifications," JHEP 04 (2000) 020, hep-th/0001132.

[47] J. Fuchs, C. Schweigert, and J. Walcher, "Projections in string theory and boundary states for Gepner models," Nucl. Phys. B588 (2000) 110-148, hep-th/0003298.

[48] C. Vafa, "Extending mirror conjecture to Calabi-Yau with bundles," hep-th/9804131.

[49] A. Strominger, S.-T. Yau, and E. Zaslow, "Mirror symmetry is T-duality," Nucl. Phys. B479 (1996) 243-259, hep-th/9606040.

[50] R. Gopakumar and C. Vafa, "Branes and fundamental groups," Adv. Theor. Math. Phys. 2 (1998) 399-411, hep-th/9712048. 